\title{
ORIGIN OF QUENCHING CRACKS
}

\author{
By Howard Scott
}

\section{ABSTRACT}

During the investigation of the dimensional changes which tool steel undergoes on hardening, it was found that a 1-inch diameter cylinder was very susceptible to cracking when quenched in oil but not when quenched in water. Presumably the stresses produced by the faster quenching (the latter case) are greater than in the former, so evidence of the cause of failure under low stress and not under high was sought. Examination of the cracked specimens showed that failure was due to tensional stress at the surface. The axial stress at the surface after quenching in water was found by experiment to be compressional and high. It was concluded therefrom that hardened steel, like glass, is highly resistant to compressional stress but not to tensional stress.

Conditions producing cracks in a particular oil-hardening steel were determined experimentally. The observations recorded show cracks are produced by permanent stress, which reaches a maximum value at ordinary temperatures. Permanent tensional stress can be produced at the surface by cooling only when the coefficient of expansion is negative and the steel is plastic. This condition was demonstrated from an experimental study of the expansion of steel during hardening.

From an analysis of the temperature distribution during quenching expressions were derived showing the relations between the major variables of quenching and the internal stress. These relations are in agreement with the experimental results and suggest practical expedients for the control of internal stress.
CONTENTS
I. Introduction
II. Experimental study of quenching cracks
III. Stress generation in cooling glass _... 409
IV. Expansion phenomena during quenching -
V. Temperature distribution during quenching
VI. Estimation of permanent stress._._.
VII. Control of quenching cracks. 434
VIII. Summary
IX. Bibliography

\section{INTRODUCTION}

Of the ills to which hardened steel is heir, perhaps, the most evasive, if not the most frequent, are quenching cracks. They appear to bear no definite relation to any of the variables of quenching except quenching temperature. We know that a high-quenching temperature is favorable to cracking, and a coarse fracture is taken 
as evidence, not to be denied, of improper heat treatment. Hence, the general rule of quenching from just above the critical range. This rule probably owes its origin more to the necessity for safety in quenching than to the physical properties obtained by its application. A low-quenching temperature does not necessarily give full hardness nor furnish maximum resistance to wear. Brayshaw, in fact, says, "*** in present-day practice the treatment required to produce the maximum cutting or wearing condition is necessarily modified to save the articles from cracking or warping." It is therefore advantageous to find means for safely quenching from high temperatures, if for no other reason than to determine the effect of quenching temperature on important physical properties.

This matter took on a more specific aspect when the writer undertook an investigation of the effect of heat treatment on certain properties of tool steel of importance in gauges. A large number of failures from cracking were observed when 1-inch diameter cylinders made from deep-hardening steels were quenched in oil, even from fairly low temperatures, but not when quenched in water. As this occurrence was too frequent to be accidental, systematic experiments were undertaken to determine the limiting conditions for safety in quenching. The results were fully confirmatory of previous experience and are given in the following section.

An adequate explanation of this phenomenon was not to be found in the literature. Brayshaw (1) ${ }^{1}$ after exhaustive experiments on the effect of initial treatment associates cracking with the prior treatment. Benedicks (2), however, points out that the benefit conferred by Brayshaw's recommended treatment, prolonged heating between $A c_{1}$ and $A r_{1}$, is gained by reducing the "hardening carbon at the final quenching." The benefit is therefore of the same nature as that conferred by a low-quenching temperature. It is, of course, obvious that the initial state of stress is immaterial for stresses of an appreciable magnitude can not exist after the quenching temperature is reached and maintained for a few minutes. Prior treatment, therefore, offers no solution to the problem.

Recognizing that cracks are primarily due to the quench, Portevin(3) attributes the type he has so carefully studied to nonuniform quenching. He notes a general relation between the cracks and soft spots in the steel revealed by hardness tests and etching. Such cracks are often associated by American metallurgists with "abnormal" steel. Portevin's work offers no explanation of cracks in uniformly and symmetrically hardened steel.

Schwinning(4) remarks in discussing Brayshaw's paper that oracks are always due to tensional stress. This significant obser-

1 The flgures given in parentheses here and throughout the text relate to the reference numbers in the bibliography given at the end of this paper. 
vation is confirmed in this report. Jasper(5) attributes such cracks to the thermal gradients which exist during quenching, but, as Honda(6) and others have found, cracks do not usually occur until the steel has reached ordinary temperatures. This observation eliminates purely thermal stresses, which disappear when the steel reaches ordinary temperatures, as the immediate cause of such cracks. In order to account for residual stresses without temperature gradients, Honda assumes that they are due to nonuniform distribution of austenite in the hardened steel. McCance(7), however, shows in his discussion of Honda's paper that this can not be the sole cause of quenching cracks. Moreover, he makes a very important observation which is fully confirmed by this work, namely, that residual stresses are reversed stresses resulting from plastic deformation. This matter is discussed by Heyn(8), but confined to casting problems. Masing(9) carries the conception somewhat farther than Heyn and establishes by mathematical analysis the possibility of computing such stresses.

Compressional surface stress has been measured in hardened steel by Jasper(5) and others. The conditions and factors which determine the sign and magnitude of the stress are, however, by no means clear from the references cited. To approach this rather complicated subject with the least danger of confusion it is well to consider first the stress situation in a relatively simple case, namely, that of cooling glass.

The extension of the laws of stress generation in cooling glass to the case of steel during quenching requires astudy of the expansion characteristics of the temperature distribution in steel during quenching. The sections devoted and the above matters are followed by a discussion of applications of the general relations deduced to practical hardening problems.

\section{EXPERIMENTAL STUDY OF, QUENCHING CRACKS}

For the study of quenching cracks solid cylinders 4 inches long and of several diameters were used. The $J$ composition of Table 1 was selected for detailed study. This is an appropriate composition, for it hardens deeply, to the center of $1 \frac{1}{2}$-inch rounds on oil quenching, and subsidiary work had already been done on it. Depth of hardening is important, for cracking in simple shapes is prevalent only when hardening is complete. The case of incomplete hardening is distinctly different and will be considered separately.

The specimens were individually suspended by wires in groups of not more than four in a horizontal electric muffle furnace through which a stream of illuminating gas was passed. After reaching the temperature indicated they were held for 15 minutes and then quenched with vigorous stirring until cold, or as otherwise noted, in 
the quenching baths selected. Four quenching baths of about 18gallon capacity each-namely, water, 60 per cent glycerin in water, neat's-foot oil, and a prepared commercial quenching oil-were chosen to show the effect of media of different cooling powers. These baths showed a fairly uniform decrease in cooling power in the order listed, as may be seen from the quenching curves of Figure 1.

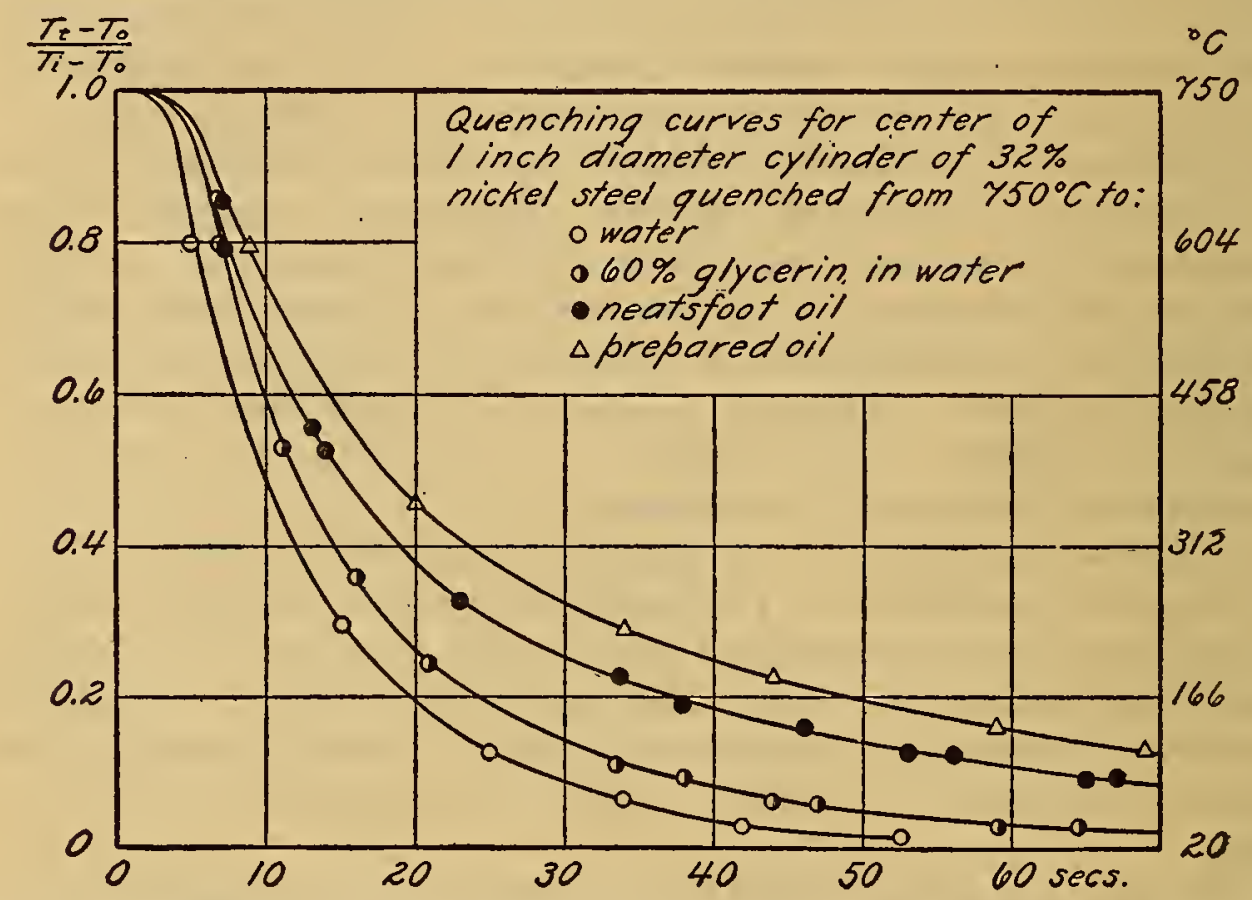

Fig. 1.-Quenching curves of the center of a 1-inch diameter cylinder, of 32 per cent nickel steel quenched in water, 60 per cent glycerin in water, neats-foot oil, and a prepared oil

The quenching curves of Figure 1 are for the center of a 1-inch diameter cylinder of 32 per cent nickel steel on quenching from $750^{\circ}$ C. The method of obtaining these curves has been described(11). Thirty-two per cent nickel steel has about the same cooling properties as the steel used here during quenching and may be quenched repeatedly without cracking.

TABLE 1.-Chemical composition of steels

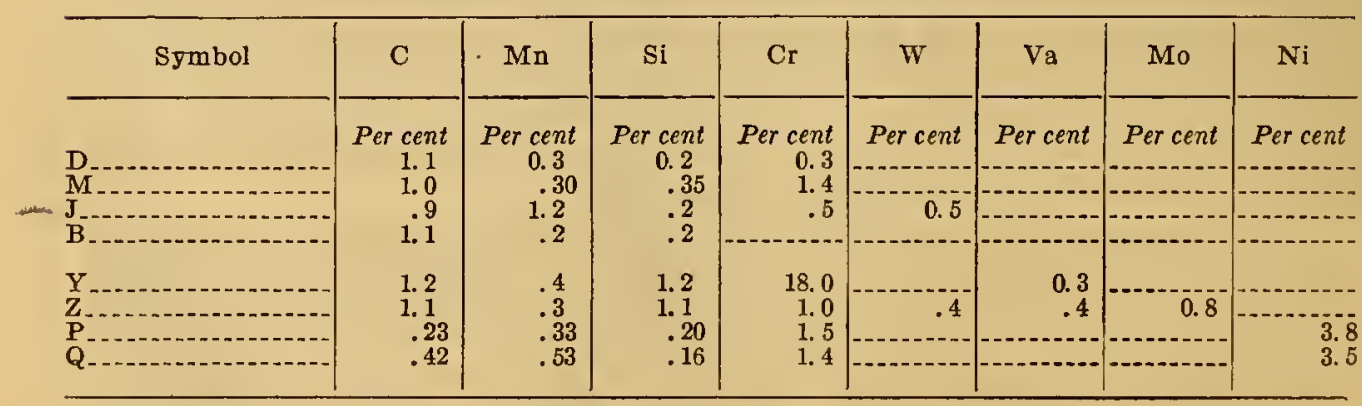


In the initial experiments three groups of four 1-inch diameter specimens were heated separately to three different temperatures and one specimen of each group quenched in each of the four media. They were then examined for cracks and, if none were found within a few days, they were deep etched in hot concentrated hydrochloric acid. The results are given in Table 2. The columns headed "as quenched" state whether or not the specimen cracked before deep etching and those headed "deep etched" state whether or not it cracked on deep etching. Classification in three groups as regards tendency to crack is, therefore, possible. In no case did a specimen crack visibly while in the bath. The nearest approach to this was a one-half inch specimen quenched in water which cracked audibly less than a minute after withdrawal. Generally the cracks (as quenched) were not found until some hours after quenching. Evidently the stress does not reach a maximum until the specimen has cooled to ordinary temperatures.

It is quite important to know whether the time elapsed between the completion of cooling and the visual detection of a crack is due to increase in the intensity of the stress with time or to progressive development of the crack from the incipient stage. Progressive growth of a crack has been observed. A short crack was located by microscopic examination a few minutes after quenching. The crack was observed as it extended throughout the full length of the specimen and widened until quite plain to the unaided eye. It is therefore probable that the stress reaches a maximum as soon as the piece is cold, but that time is required for the development of cracks to visual size by tearing of the metal.

Of the 1-inch specimens, all of those quenched in either of the oils cracked either as quenched or after deep etching. Moreover, they cracked as quenched, except with the lowest quenching temperature. Two specimens cracked on quenching in the glycerin-water solution, but none cracked on water quenching even on deep etching. These results confirmed previous incidental observations but are of such radical nature as to demand further investigation.

If specimens of 1-inch diameter crack on quenching in oil, it should be possible to crack specimens in water also by taking a small enough diameter to give the same temperature distribution as on oil quenching. To test this proposition and extend the experiments over a greaterer range of quenching conditions than those of the initial experiments, specimens of $1 / 2,3 / 411 / 2$, and 2 inches diameter and 4 inches long were prepared. These were quenched as were the 1-inch diameter specimens. A quenching temperature of $850^{\circ} \mathrm{C}$. was used in order to obtain definite effects. The results are given in Table 2. 
TABLE 2.-Observations on quenching cracks in solid cylinders 4 inches long EFFECT OF QUENCHING TEMPERATURE

\begin{tabular}{|c|c|c|c|c|c|c|c|c|c|}
\hline \multirow{2}{*}{$\begin{array}{c}\text { Quenching } \\
\text { temperature, } \\
{ }^{\circ} \mathrm{C} .\end{array}$} & \multirow{2}{*}{$\begin{array}{l}\text { Diam- } \\
\text { eter }\end{array}$} & \multicolumn{2}{|c|}{ Prepared oil } & \multicolumn{2}{|c|}{ Neat's-foot oil } & \multicolumn{2}{|c|}{60 per cent glycerin } & \multicolumn{2}{|c|}{ Water } \\
\hline & & $\underset{\text { quenched }}{\text { As }}$ & $\begin{array}{l}\text { Deep } \\
\text { etched }\end{array}$ & $\underset{\text { quenched }}{\text { As }}$ & $\begin{array}{l}\text { Deep } \\
\text { etched }\end{array}$ & $\begin{array}{c}\text { As } \\
\text { quenched }\end{array}$ & $\begin{array}{l}\text { Deep } \\
\text { etched }\end{array}$ & As & $\begin{array}{l}\text { Deep } \\
\text { etched }\end{array}$ \\
\hline $780 \ldots$ & $\begin{array}{c}\text { Inches } \\
1\end{array}$ & No ${ }^{1}$ & Yes _ & No....... & Yes_. & No. & No. & No... & No. \\
\hline $820 \ldots$ & 1 & Yes.. & $\ldots$ & Yes.. & & No. & $\left\{\begin{array}{l}\text { No- } \\
\text { Yes }\end{array}\right.$ & Wo....... & No. \\
\hline $850 \ldots \ldots$ & 1 & Yes.. & $\ldots$ & Yes.- & $\cdots$ & No_...... & Yes.... & No...... & No. \\
\hline
\end{tabular}

EFFECT OF DIAMETER

\begin{tabular}{|c|c|c|c|c|c|c|c|c|c|}
\hline $\begin{array}{l}850 \\
850 \\
850 \\
850 \\
850\end{array}$ & $\begin{array}{l}1 / 2 \\
3 / 4 \\
1 \\
11 / 2 \\
2\end{array}$ & $\begin{array}{l}\text { No...... } \\
\text { Yes..... } \\
\text { Yes..... } \\
\text { No....... } \\
\text { No....... }\end{array}$ & \begin{tabular}{|l|} 
No \\
Yes \\
No \\
\end{tabular} & \begin{tabular}{l} 
No...... \\
Yes..... \\
Yes $\ldots \ldots-$ \\
Yes 2.... \\
\hdashline
\end{tabular} & 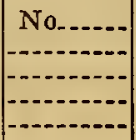 & \begin{tabular}{l} 
Yes..... \\
No._.... \\
No...... \\
No....... \\
\hdashline
\end{tabular} & 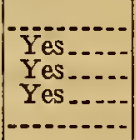 & \begin{tabular}{|l|} 
Yes \\
No...... \\
No.....- \\
No...... \\
No......
\end{tabular} & $\begin{array}{l}\text { Yes. } \\
\text { No. } \\
\text { No. } \\
\text { No. }\end{array}$ \\
\hline
\end{tabular}

1 "Yes" and "No" answers question as to whether cylinders cracked or not.

2 specimen dropped in bath before cold.

3 specimen did not harden in center.

The one-half inch specimens behaved just the reverse of the 1-inch ones; they cracked as quenched in water, but not as quenched or on deep etching following oil quenching. Deep etching developed many shallow grooves on the surface but, lacking depth, they were not classified as cracks. Deep-etching specimens of different size furnishes comparisons of doubtful value; the results are given for what they may be worth. The three-fourths-inch specimens appear to be the most susceptible to cracking of all the sizes used. They cracked as quenched in oil and on deep etching after quenching in water and in the glycerin-water solution.

The specimens larger than 1 inch behaved much the same as the 1-inch, with a significant exception. The 2-inch specimen quenched in oil did not crack before or after deep etching. This observation would be inconsistent with the rest were the specimen completely hardened. To determine whether or not hardening was complete, it was sectioned midway between the ends and the section explored with the Rockwell hardness tester. A soft area (45 Rockwell diamond cone) was found near the center. As the soft area was small, it is probable that the $11 / 2$-inch specimen hardened completely. On the basis of this observation, the 2-inch specimen must be classified with surface-hardened steels, the stress situation in which will be discussed shortly.

It is now well to make some general observations regarding the occurrence of cracks before taking up specific aspects. The most important of these observations is that all cracks noted above were due to tensional stress at the surface. Cracks due to surface tension can generally be identified by the fact that they are wider at the sur- 
face than at the center. Brittle materials, such as glass and tool steel, are much less resistant to tensional then to compressional stress. This is particularly true when the stress is tensional at the surface where discontinuities can not be avoided and accounts in part for the failures observed. This observation suggests also the reason for the failure of large specimens quenched in water to crack when the stress would be expected to be greater than on oil quenching. If the stress is compressional at the surface, it will be balanced by tensional stress at the center, where much greater stress can be sustained without failure.

Of the many cracked specimens examined, only one positive example of failure due to tension at the center (compression at the surface) was observed. A three-fourths-inch diameter plug gauge quenched in water failed during lapping. The fracture near the surface was fresh, but discolored near the center. Evidently the crack started from the center holes and was completed by the slight external stress incident to machining. In this case the stress at the center was tensional and the crack naturally started there. It may therefore be concluded that tensional stress is the primary source of quenching cracks and that its effects are most severe at the surface.

More direct evidence of compressional stress at the surface of large cylinders quenched in water can be gained only by stress measurements. Stress measurements have been made on a few specimens by the method of Heyn(8). Tensional surface stress can not be detected by this method with any certainty, for if it is of sufficient magnitude to give definite results the specimen will probably crack. The axial stress is measured by the change in length on grinding successive peripheral layers off a cylinder. The length change is related to the unit stress in the $N$ th layer by the formula:

$$
F_{\mathrm{n}}=\frac{D_{\mathrm{n}}^{2}\left(l_{\mathrm{n}}-l_{\mathrm{o}}\right)+D_{\mathrm{n}-1}^{2}\left(l_{\mathrm{n}-1}-l_{\mathrm{o}}\right)}{D_{\mathrm{n}-1}^{2}-D_{\mathrm{n}}^{2}} \cdot \frac{E}{l^{\prime}}
$$

Here $D_{\mathrm{n}}$ is the diameter and $l_{\mathrm{n}}$ the length when the $n$th layer is removed, $l_{0}$ the initial length, $l^{\prime}$ the length of the layer removed, and $E$ the modulus of elasticity.

Figure 2 shows the unit axial stress estimated as indicated above, using $E=30,000,000 \mathrm{lbs}$./in ./in., and plotted against $\left(\frac{r}{c}\right)^{2}$ where $r$ is the distance from the center and $c$ the radius. The positive (tension) areas and the negative (compression) areas must then balance. All three specimens are 1-inch diameter, 4 inches long, quenched in water and ground over a 3-inch length. M30 and M32 are of different composition from that used in the quenching experiments (Table 1), but are of a deep-hardening composition which behaves $50351^{\circ}-25 \dagger-2$ 
very much the same. The stress is compressional near the surface and tensional farther in, but low near the center.

D10 of Figure 2 is a surface-hardening steel of the composition given in Table 1, which hardens to a depth of about $1 / 8$ inch on water quenching the 1-inch round. The surface of this specimen was also under compressional stress, but it is very much higher than in the deep-hardening steel. This is due to the fact that the martensite shell has a greater specific volume than the soft core. This difference in specific volume due to incomplete hardening dominates the stress effects in such steels and undoubtedly prevented the

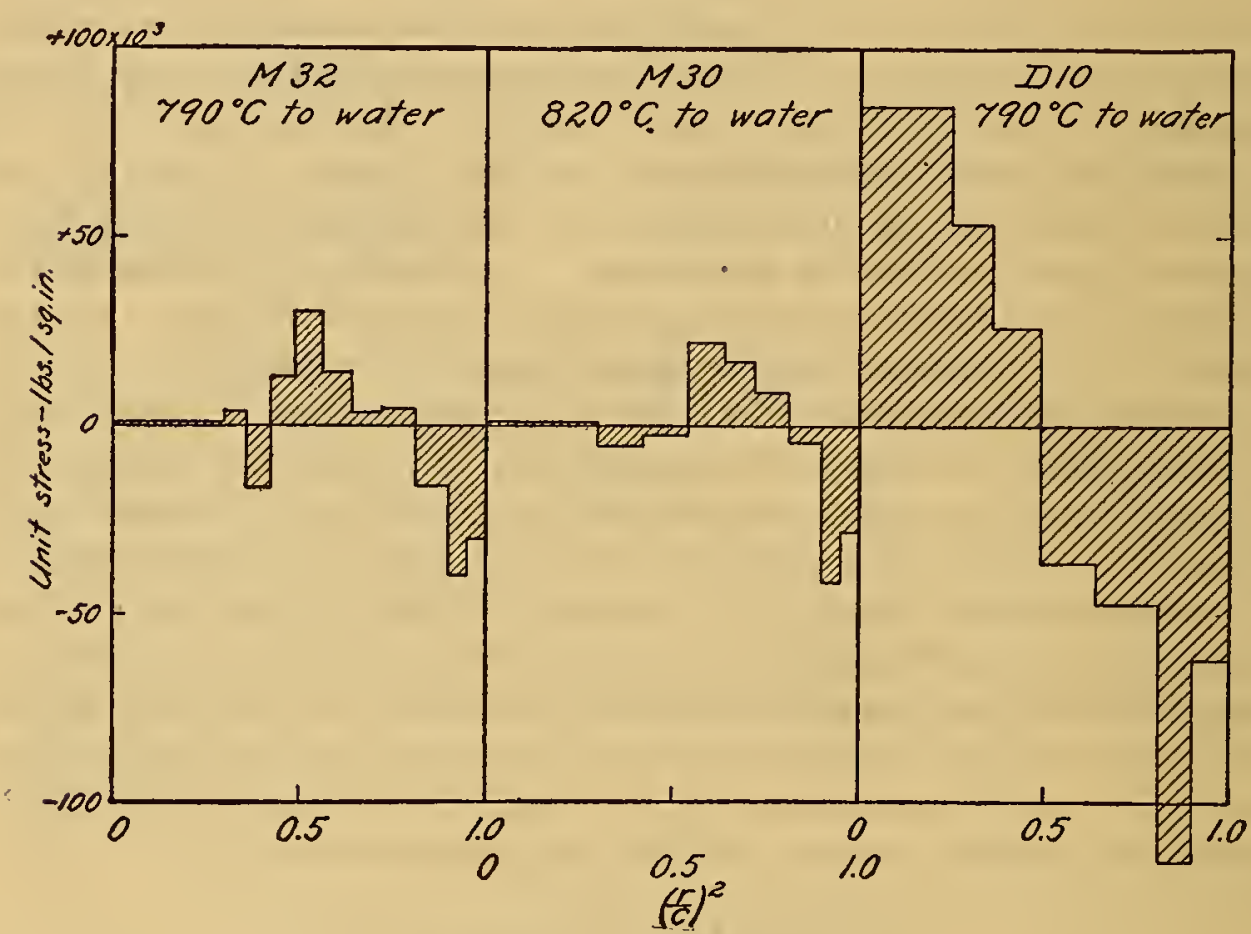

Frg. 2.-Unit axial stress in cylinders 1 inch diameter and 4 inches long measured by grinding off peripheral layers 3 inches long and plotted against the square of the ratio of radius as ground to initial radius. Positive stress is tension and negative stress compression

generation of tensional stress at the surface of the 2-inch diameter cylinder quenched in oil.

Jasper (5) has also observed compressional stress at the surface of steel cylinders, which, from their composition and treatment, must have been hardened on the surface only. He notes also the effect of tempering and of alternating stress in reducing the internal stress.

Having thus associated quenching cracks with tensional surface stress and shown that deep-hardening steels rapidly quenched in large sizes are under compressional surface stress, the occurrence of cracks can be studied more intelligently. Photographic recording 
of the cracks observed is in many cases unsatisfactory, because cracks of different nature sometimes occur on opposite sides of some specimens. There is always a longitudinal crack and often circumferential cracks on the opposite side. This is illustrated by Figure 3. The lines representing cracks are tracings of the cracks found on the peripheral surface after deep etching. In practically all cases of cracking as quenched the cracks were longitudinal. That the cracks depart in some cases from the longitudinal direction when developed by deep etching is, perhaps, significant.

Location of cracks on periphery of quenched

steel cylinders after deep-etching
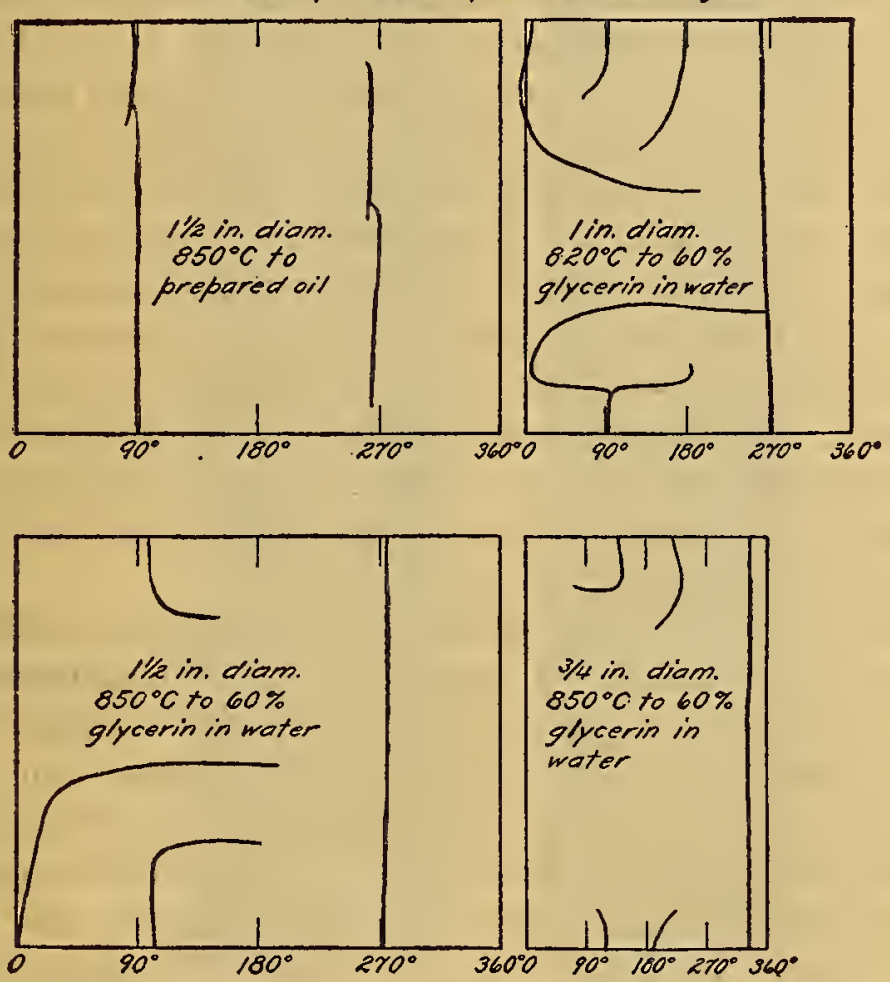

FIG. 3.-Tracings of cracks found on the peripheral surface of quenched cylinders after deep etching

A number of failures due to cracking have been observed in more complicated shapes and in other compositions than considered above. Representative examples are shown in Figures 4 and 5. Figure 4 shows two 1-inch cylinders of the $M$ composition which were slotted with a hack saw along the axis for half the length. One was then quenched in oil $(a)$ and the other in water $(b)$. The former did not crack, but the latter did at the bottom of the saw cut. The presence of the crack was not suspected until the failure of the specimen to ring when hit was noticed. This is a reversal of the findings for solid cylinders otherwise treated the same. It signifies that pieces 
having interior surfaces not reached by the coolant will not behave the same as pieces of the same external dimensions but solid.

Figure 5 shows some cracks found in plug gauges; $(a)$ is the only example of cracking due to surface compression so far found and already discussed. The photograph was taken after the fresh fracture at the edge had become soiled, but its outline may still be traced; $(b)$ is the same steel and shape quenched in oil which cracked from tension at the surface; $(c)$ and $(d)$ are gauges having a threeeighths-inch handle and three-fourths-inch body. One cracked in the body on oil quenching $(c)$, and the other in the handle on water quenching $(d) ;(e)$ is a three-eighths-inch diameter gauge which cracked on water quenching. These gauges represent several widely different compositions and departures in form from the standard cylinders, yet the results are what would be expected from Table 2 .

The discussion so far has been confined to one type of crack-a deep-seated crack which follows the general contour of the specimen. In specimens of complicated shape another type, no less objectionable, is also prevalent. Figure 6 gives some examples of this type. The cracks are local and associated with sharp changes in contour; (a) shows a circumferential crack where the handle joins the body of a plug gauge. The handle has been broken off flush with the body to give an unobstructed view. These cracks are inclined at an angle to the axis of the gauge, indicating an attempt on the part of the gauge to relieve its sharp outlines.

A similar type of fracture is produced in a distinctly different manner which may be indicative of its cause. One-inch diameter cylinders having an 0.2-inch diameter hole drilled axially through them were fitted with disk caps $1 / 4$ inch thick and 1 inch diameter on both ends. On water quenching these specimens cracked circumferentially, but only under the caps (fig. $6(b)$ and $(c)$ ). These observations appear to be quite significant but have not been followed up. They are introduced chiefly to show that the deep-seated cracks are not the only type encountered, although the present paper is confined to them.

The preceding observations on deep-seated quenching cracks may now be summarized as follows:

1. Cracking is produced by tensional stress and is most prevalent when the stress is tensional at the surface.

2. The stress-producing cracks in cylinders is tangential.

3. Cracks do not usually develop to risual size until some time after the steel reaches ordinary temperatures throughout.

4. The time element in cracking is due to the gradual growth of the crack rather than the stress.

Tensional surface stress of a magnitude sufficient to crack is produced in simple solid shapes of completely hardened steel only when 
Scientific Papers of the Bureau of Standards, Vol. 20

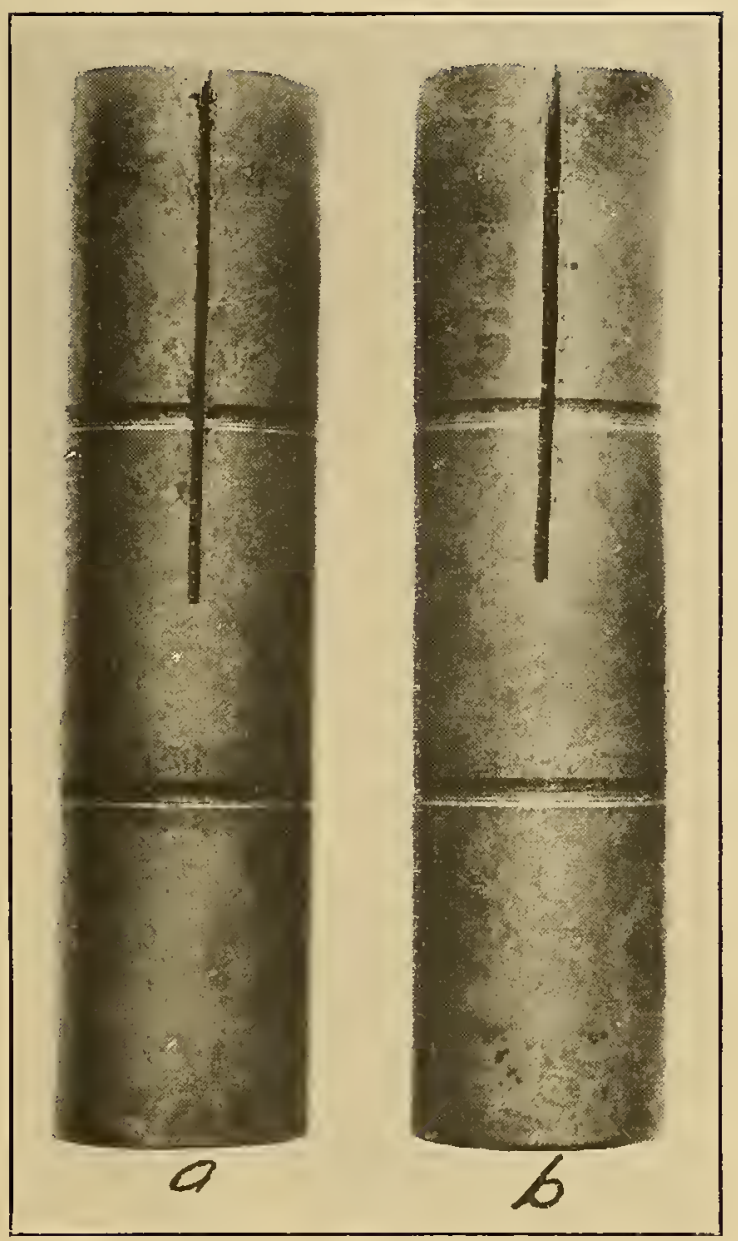

FIa. 4.--Reversal of quenching cracks caused by slotting specimens, composition $M$, quenched from $850^{\circ} \mathrm{C} . ;(a)$ to oil, (b) to water

$(b)$ is cracked at the bottom of the slot. $\frac{15}{10}$ size 
Scientific Papers of the Bureau of Standards, Vol. 20

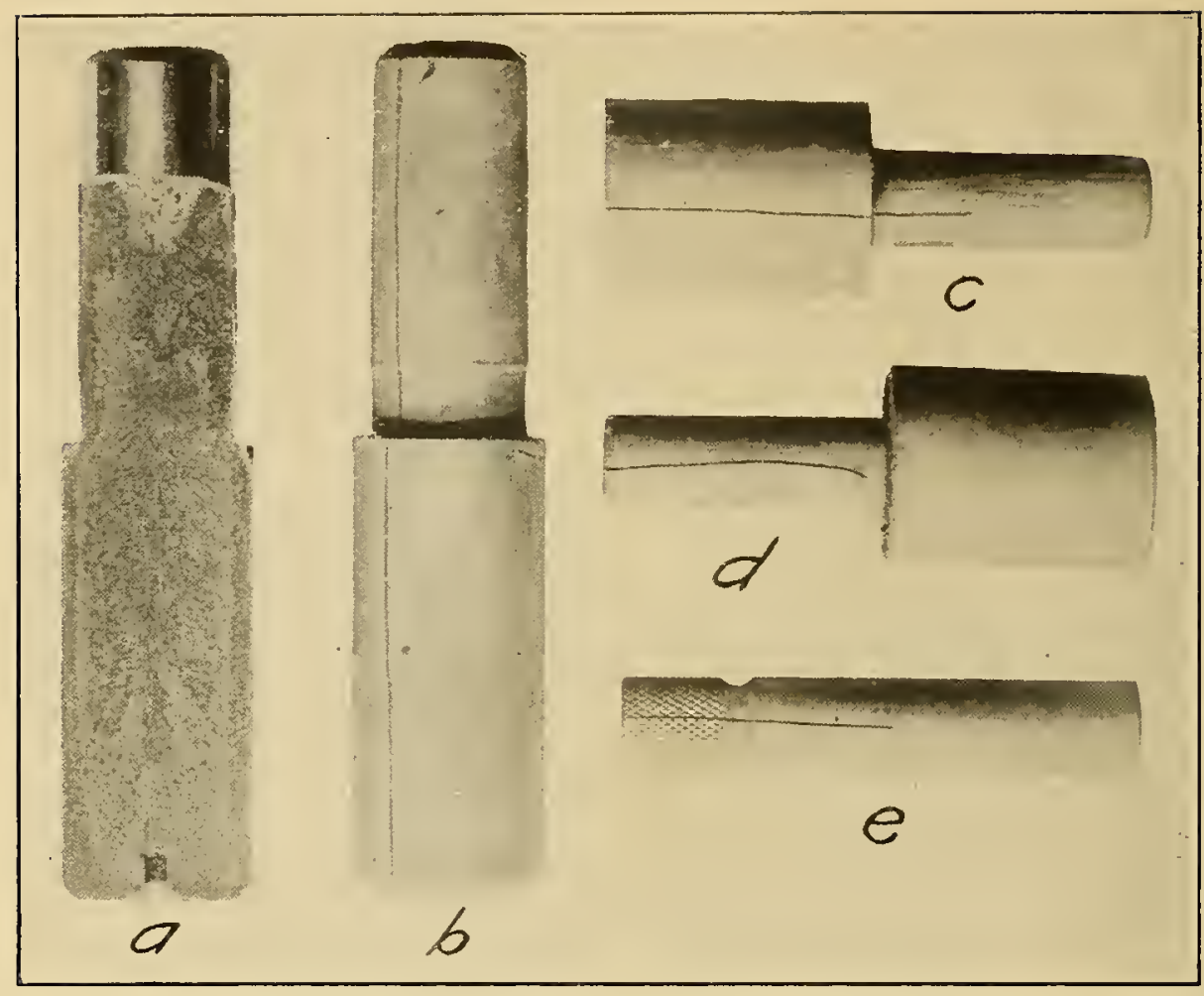

FIG. 5.-Represenlative eracks in plug gauges

$(a),(b),(c)$, and (e), ehrome-bearing steel, composition Mi; (d) earbon tool steel, composition B. $(a)$ was quenched in water from $820^{\circ} \mathrm{C}$., (b) and $(c)$ in oil from $840^{\circ} \mathrm{C} .,(d)$ in water from $820^{\circ} \mathrm{C}$., and $(e)$ from $810^{\circ} \mathrm{C}$. All except $(a)$ deep-etched to develop cracks already present. (a) Cracked from tensional stress at the eenter, the edges failing last; (b), (c), (d), and (c), from tensional stress at the surface. $\frac{9}{10}$ size

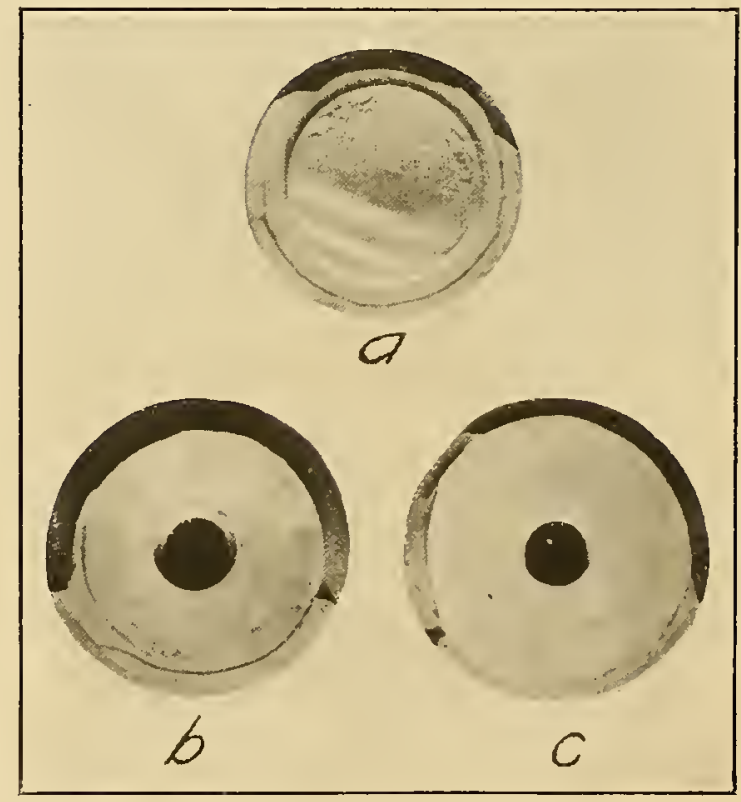

Fig. 6.-Local cracks

(a) Plug gange $7 / 8$ inch diameter in body and $5 / 8$ inch diameter in handle, composition $\mathrm{X}$ quenehed in water fron $810^{\circ} \mathrm{C}$. Handle broken off to afford view of circumferential eracks where body joins handle. (b) ancl (c), standard specimens with ecntral hole quenehed in water with eaps on ends. (b) is M composition anenehed from $820^{\circ} \mathrm{C}$. and (c) D composition quenched from $800^{\circ} \mathrm{C}$. All slightly etehed 
the cooling rate is slow and the diameter moaerate ( 1 inch diameter quenched in oil) or when the diameter is small and the cooling fast (one-half inch diameter quenched in water). It is not of dangerous magnitude if the diameter is small and the cooling rate slow. A surface not effectively reached by the quenching medium may modify these effects.

Fast quenching of large sections or surface hardening produces compressional stress at the surface. If deep-seated cracks then occur, they start at the center where the stress is tensional. However, the greatest danger with high compressional surface stress is from local cracks associated with abrupt changes in the surface contour.

These facts reveal a reversal in sign of the surface stress with increasing size or quenching power of the bath. Before the reversal there is a dangerous range of sizes or cooling powers in which range the tensional stress at the surface is sufficiently high to cause cracking under the experimental conditions. The problem, however, is not how to crack steel but how to avoid cracking it when the sizes come within the dangerous zone with an otherwise appropriate quenching medium. This is a matter which demands study of the basic causes of stress generation. The explanation developed must account for or be compatible with the above conclusions. Such an explanation can be developed from the laws of stress generation in cooling glass, and the following pages are devoted to them.

\section{STRESS GENERATION IN COOLING GLASS}

Internal stress in a solid is due to relative elastic deformation of its component elements. Differential deformations may be produced by constitutional differences, as in surface hardened steels, or by temperature differences in a body having a finite coefficient of expansion. The latter case is of particular interest here. If a body having a positive coefficient of expansion (increase in volume with increasing temperature) be heated at a uniform rate, the surface will have a greater specific volume than the center, and consequently will be under compression while the temperature difference is maintained. During cooling the temperature difference will be in the opposite direction, so the stress at the surface will be tensional. In either case the stress will disappear as soon as heating or cooling is stopped, for the temperature differences will then vanish. Because of its transient nature such stress is called temporary stress. As the temporary stress is nil at ordinary temperatures, it can not be the cause of the quenching cracks described in the preceding section. Hence, it remains to be determined how stresses can be developed which are at maximum values when no temperature differences exist.

Suppose a temperature difference established by cooling an elastic body without the development of stress. Removal of this tempera- 
ture difference will then cause the center to shrink reiative to the surface, so the surface will be under compression when cooling stops. This stress is the reverse of the temporary stress produced by cooling. It is called permanent stress, because it does not depend on a transient temperature difference for its existence. Such a stress can be produced in a body like glass which is plastic at high temperatures and elastic at low temperatures.

The above facts are illustrated graphically for the case of cooling glass by Figure 7 . In this figure the ordinates represent temperature and stress and the abscissas thickness of a plate. It is assumed

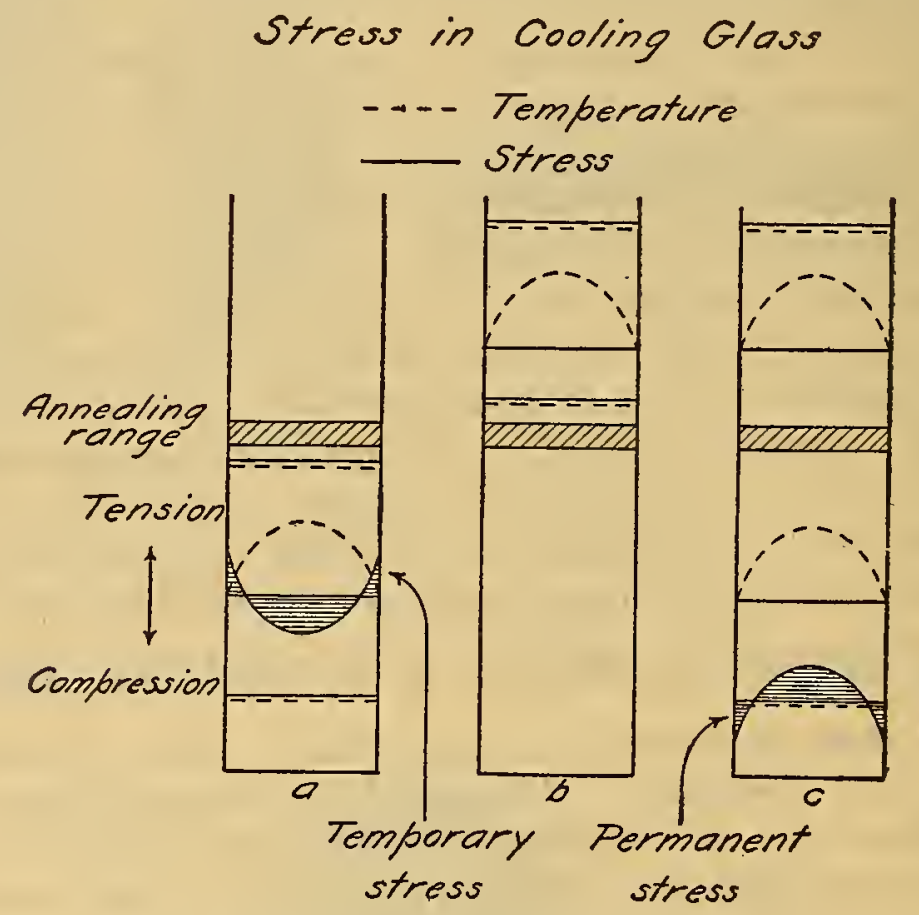

FIG. 7.-Relation between stress and temperature distribution in slabs of glass cooling linearly

The glass is assumed to be plastic above the annealing range and elastic below it

that the glass is plastic; that is, flows under any appreciable stress, when above the annealing range and is elastic to the extent deformed below it. Also the cooling is assumed to be linear and the coefficient of expansion positive and constant.

The first case (a), of Figure 7, represents the condition for the generation of temporary stress and requires no further comment. The second case $(b)$ represents the effect of temperature differences above the annealing range. Obviously, no stress can exist there either during cooling or when cooling is stopped. The third and most important case is that of permanent stress $(c)$. Here a temperature difference is imposed above the annealing range and maintained below it. No stress is developed so long as the difference is maintained, even below the annealing range, but when cooling is stooped 
at some temperature below the annealing range a stress is developed as the temperature difference disappears. The final stress is equal to but opposite in sign from the temporary stress which would be induced by the same temperature distribution. The permanent stress so developed reaches a maximum only when the temperature difference is completely dissipated.

Figure 7 applies only to linear cooling. When rapid cooling is involved, as on quenching steel, the temperature differences decrease about as the temperature. This will modify the situation depicted by Figure 7. The stress as before will be zero when the annealing range is entered, but now permanent stress will be developed as the temperature differences drop below those existing when the annealing range was entered. Evidently the final permanent stress will be just the same as that produced by a linear cooling rate which gives the same temperature difference as variable cooling does at the time of entering the annealing range.

This condition introduces a new difficulty. As the temperature difference decreases during cooling, the difference will be less when the center becomes elastic than when the surface became elastic. However, when the surface becomes elastic, the permanent stress is fixed for further plastic deformation and is then inbibited. It therefore follows that the permanent stress in a solid body is determined by the temperature distribution when the surface becomes elastic. This conclusion is the basis for the estimation of permanent stress.

Having found that the sign of the surface stress is the chief criterion of whether steel will crack or not, it is well to tabulate the sign of the stress, tension for positive stress, and compression for negative stress for important cooling conditions as in Table 3. A new condition is added, namely, that of a negative coefficient of expansion. Reversing the sign of the coefficient reverses the stress, other things the same. This case is added, for the coefficient of expansion of steel during quenching is negative over a certain temperature range. In fact, from Table 3, the permanent tensional surface stress observed experimentally could not be developed by quenching were not the coefficient negative at some time during quenching. With due regard to previous developments, the important conclusion can now be drawn that the negative coefficient of expansion during quenching is the major cause of quenching cracks.

TABLE 3.-Conditions required to produce tension or compression, either temporary or permanent, at the surface of solid bodies during heating or cooling

\begin{tabular}{|c|c|c|c|}
\hline Coefficient of expansion & Direction of heat flow & Temporary stress & Permanent stress \\
\hline Positi & $\left\{\begin{array}{l}\text { In }_{\text {Out }} \\
\text { - }\end{array}\right.$ & \multirow{2}{*}{$\begin{array}{l}\text { Compression } \\
\text { Tension- } \\
\text { Compression }\end{array}$} & \multirow{2}{*}{$\begin{array}{l}\text { Tension. } \\
\text { Compression. } \\
\text { Tension. }\end{array}$} \\
\hline Negative & & & \\
\hline
\end{tabular}


The conception of stress generation in cooling glass outlined above is taken from Adams and Williamson's (10) paper on the annealing of glass. These authors give an explanation of stress generation essentially as stated here. The principles have been successfully applied to the commercial annealing of optical glass and should be applicable as amplified to the hardening of steel also. But before considering further the case of steel it will be profitable to follow these authors further in the analysis of stress distribution under relatively simple conditions.

The cylinder is the most convenient form for experimental study. Adams and Williamson give the equations for the stress in this shape. It is only necessary to consider one case of Table 3 , for it is immediately convertible by suitable change of sign to any other case of this table. They give for the axial, radial, and tangential stresses at points remote from the ends of a long cylinder the following expressions:

$$
\begin{aligned}
& F_{a}=\frac{1}{8} k p c^{2} . \quad 2\left[1-\frac{2 r^{2}}{c^{2}}\right] \\
& F_{r}=\frac{1}{16} k p c^{2} .\left[1-\frac{r^{2}}{c^{2}}\right] \\
& F_{t}=\frac{1}{16} k p c^{2} . \quad\left[1-\frac{3 r^{2}}{c^{2}}\right]
\end{aligned}
$$

where $r$ is the distance from the center, $c$ the radius, $p$ the rate of temperature change taken positive for heating and negative for cooling, and $k$ a constant defined by:

$$
k=\frac{\alpha}{a^{2}(e-f)}
$$

Here $\alpha$ is the coefficient of expansion, $a^{2}$ the diffusivity, and $(e-f)$ elastic constants of the material determined by the modulus of elasticity and Poisson's ratio. As $\frac{F}{k p c^{2}}$ depends only on the ratio ${ }_{c}^{r}$, we can plot this measure of the stress against $\frac{r}{c}$ and see immediately the relations between the component stresses without bothering with the evaluation of the constants. This has been done in Figure 8 . Positive stress is tensional and negative compressional.

Figure 8 shows that the axial and tangential stresses are equal at the surface of cylinders but that the axial stress decreases inward more rapidly than the tangential. The tangential stress is therefore greater than the axial over a finite area near the surface. This explains the marked tendency of cylinders to crack longitudinally; that is, from tangential stress. Also when circumferential cracks 
are observed as quenched, they are not so deep as the longitudinal ones.

As $\frac{F}{k p c^{2}}$ is a function of $\frac{r}{c}$ alone, the stress at any point is directly proportional to the cooling rate and to a constant minus the square of its distance from the center divided by the radius.

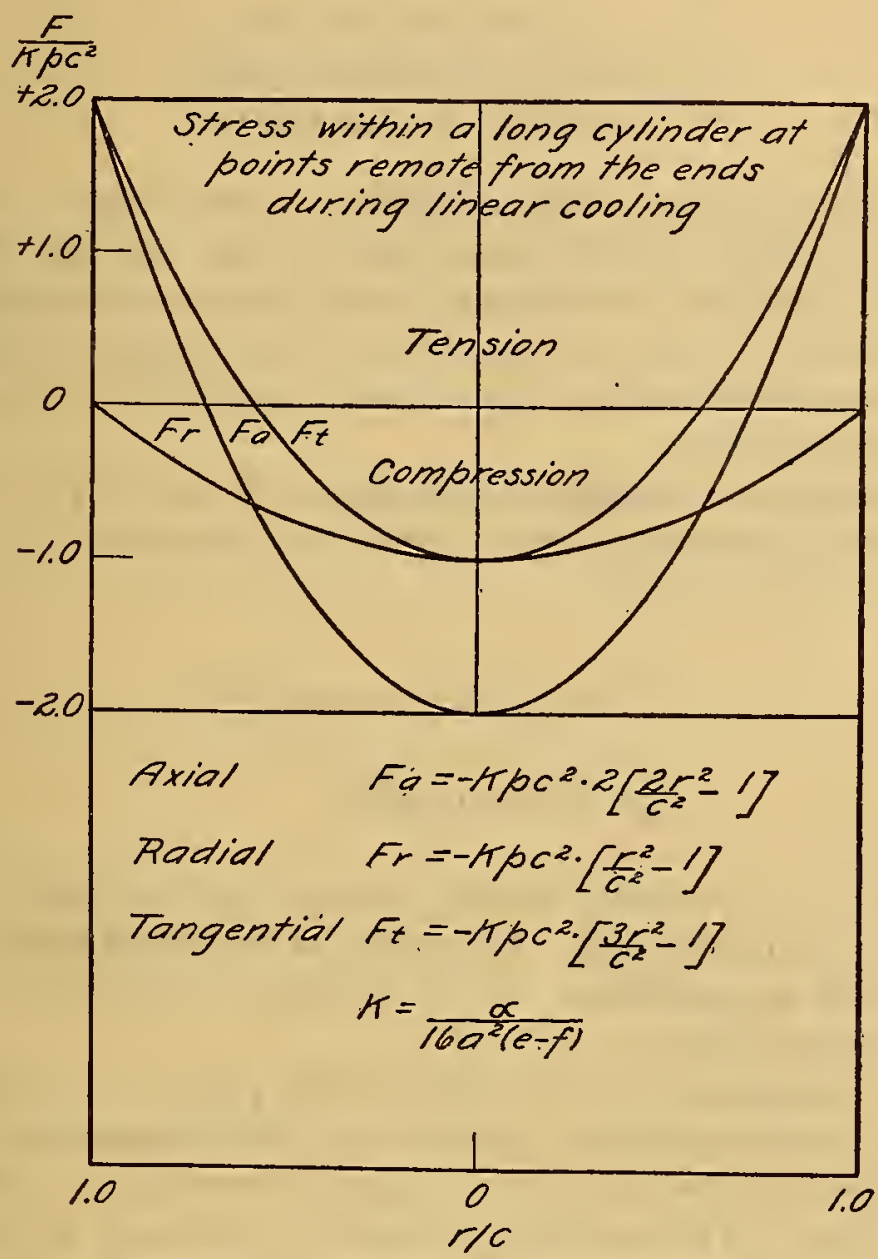

Fig. 8.-Axial $\left(F_{a}\right)$, radial $\left(F_{r}\right)$, and tangential $\left(F_{\imath}\right)$, stresses in a long cylinder during linear cooling

It is of some interest to examine the expressions given by these authors for other simple shapes. In the flat slab cooled on both surfaces:

and in the sphere:

$$
F=\frac{1}{6} \quad k n c^{2} \cdot\left[1-\frac{2 r^{2}}{c^{2}}\right]
$$

$$
\begin{aligned}
& F_{r}=\frac{1}{15} \quad k p c^{2} \cdot\left[1-\frac{r^{2}}{c^{2}}\right] \\
& F_{t}=\frac{1}{15} \quad k p c^{2} \cdot\left[1-\frac{2 r^{2}}{c^{2}}\right]
\end{aligned}
$$

Evidently the stress situation is more complicated in the cylinder than in either the slab or the sphere. 
A comparison of the surface stresses in these shapes reveals the relative magnitude of the stresses at the surface with the same cooling rate. At the surface the radial stress is zero, so only the axial and tangential stresses need be considered and they are the same at the surface of these shapes. From the above equations:

$$
\frac{F}{k p c^{2}}\left\{\begin{array}{l}
=0.167 \text { for the slab, } \\
=0.125 \text { for the cylinder, } \\
=0.067 \text { for the sphere. }
\end{array}\right.
$$

The magnitude of the surface stress in these shapes of the same diameter or thickness and cooling rate is then in the order listed. This relation will not be obtained with the same quenching media. As the quenching media is a more useful and practical variable than cooling rate, expressions for the stress in terms of the cooling media will be developed later.

If it be desired to estimate actual stresses from the above relations, the constants appearing therein must be evaluated. (e-f) may be found from the following:

$$
\begin{aligned}
& \frac{1}{e}=\text { modulus of elasticity, } \\
& \frac{f}{e}=\text { Poisson's ratio. }
\end{aligned}
$$

The diffusivity, $a^{2}$, is the thermal conductivity divided by the density times the specific heat. Its value for hardened steel is between 0.05 and 0.10 in egs units. It will be high in low-alloy steels and low in high-alloy steels.

In these considerations the end effects present in cylinders and slabs have been neglected. Adams and Williamson(10) show that the end effects do not modify seriously the stress at points remote from the edges. This analysis is therefore confined to the stress at such points. The stress distribution is, of course, greatly modified as the ends are approached; for example, the axial stress goes to zero at the end surface of a cylinder. No attempt is made to analyze the very complicated stress distribution around the ends. The cracks identified with end effects are styled local cracks. It is assumed that the stresses causing them will be less severe, the lower the maximum stress at points remote from the ends.

To pursue this subject to more useful ends, we must now consider the expansion characteristics of steel and the temperature distribution in steel as a function of the quenching media, size of specimen, and other factors. This accomplished, a more specific picture of the factors governing quenching stresses can be drawn. 
Scientific Papers of the Bureau of Standards, Vol. 20

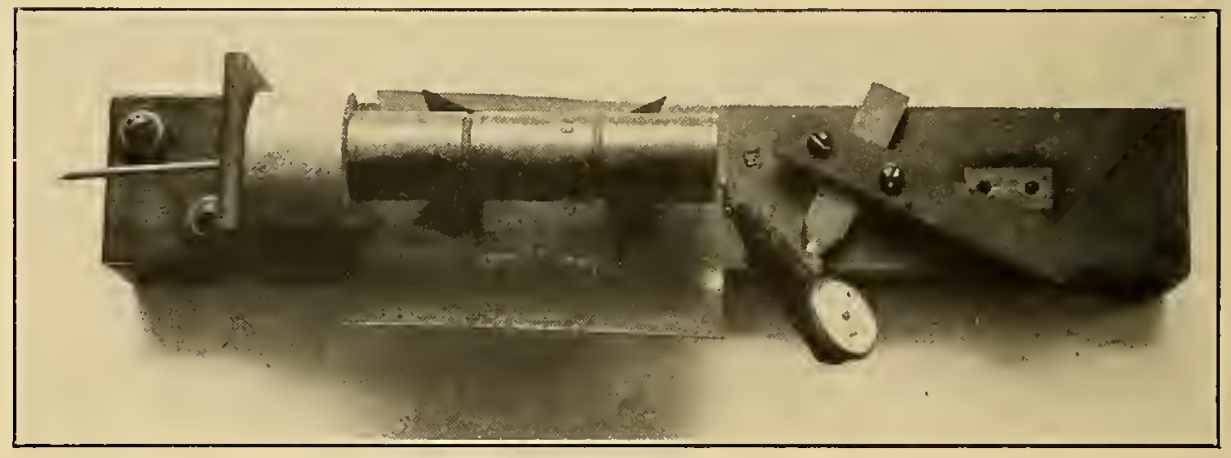

FiG. 9.-Expansion apparatus 


\section{EXPANSION PHENOMENA DURING QUENCHING}

It is well known from the work of Chevenard(12) and others that when steel hardens it undergoes a low-temperature transformation, designated $A r^{\prime \prime}$, with a marked increase in volume. During cooling down to this change the steel is austenitic and shrinks according to the coefficient of expansion of this constituent. Souder and Hidnert(13) have determined the coefficient of expansion of a number of steels above the critical range-that is in the austenitic condition-and give the average value of 0.000023 for it. This value also represents approximately the contraction down to $A r^{\prime \prime}$. The coefficient for annealed steels is much smaller, about 0.000014 , so for the steel to return to its original volume on hardening it must expand by an amount greater than that given by the difference between these coefficients times the temperature range from $A r^{\prime}$ to $A r^{\prime \prime}$. Thus, the expansion at $A r^{\prime \prime}$ is greater the lower the temperature of this transformation. More specific information on the temperature and other characteristics of this change in the oil-hardening tool steels is not available in the literature. For this reason the length changes during $A r^{\prime \prime}$ were determined experimentally.

The device used for measuring the expansion is very simple. A rigid stand was constructed on which a standard cylindrical specimen 1 inch diameter and 4 inches long with a central hole $1 / 8$-inch. diameter and 2 inches deep can be placed quickly, with the hole end in contact with a solid block of low-thermal conductivity and the other end in contact with a mechanical length indicator reading to thousandths of an inch (fig. 9). Specimens of oil-hardening steel were quenched in oil but removed from the oil before $A r^{\prime \prime}$ was reached, then placed on the stand, a thermocouple inserted in the hole, and cooled before an electric fan. The temperature and length change were measured simultaneously and periodically until cold. The results are given in the form of curves (figs. 10,11, and 12) and the compositions of the steels used in Table 1. Measurements of the length were made before and after test, so the change from the initial length could be plotted as in Figures 10 and 11. Figure 12 gives the change from the final length. The length changes are given in thousandths of an inch per inch.

Figure 10 shows the effect of quenching temperature on the hardening transformation $A r^{\prime \prime}$ of the steel $J$ used for the study of quenching cracks. Increasing the quenching temperature lowers $A r^{\prime \prime}$ and increases the magnitude of the expansion; raising the quenching temperature from 780 to $850^{\circ} \mathrm{C}$. lowers $A r^{\prime \prime}$ by $50^{\circ} \mathrm{C}$. at the start. The coefficient of expansion preceding $A r^{\prime \prime}, \alpha_{1}$, and that during the initial stages of $A r^{\prime \prime}, \alpha_{2}$, is quite important. Linear expansion curves for $\alpha_{1}=+0.000020$ and for $\alpha_{2}=-0.000040$ are 
drawn and represent approximately the expansion in the regions indicated.

In Figure 11 the effect of quenching temperature on a composition $Z$ having a high carbon and hence high free carbide content is shown. Here $A r^{\prime \prime}$ is markedly lowered, $130^{\circ}$ C. at the start, by increasing

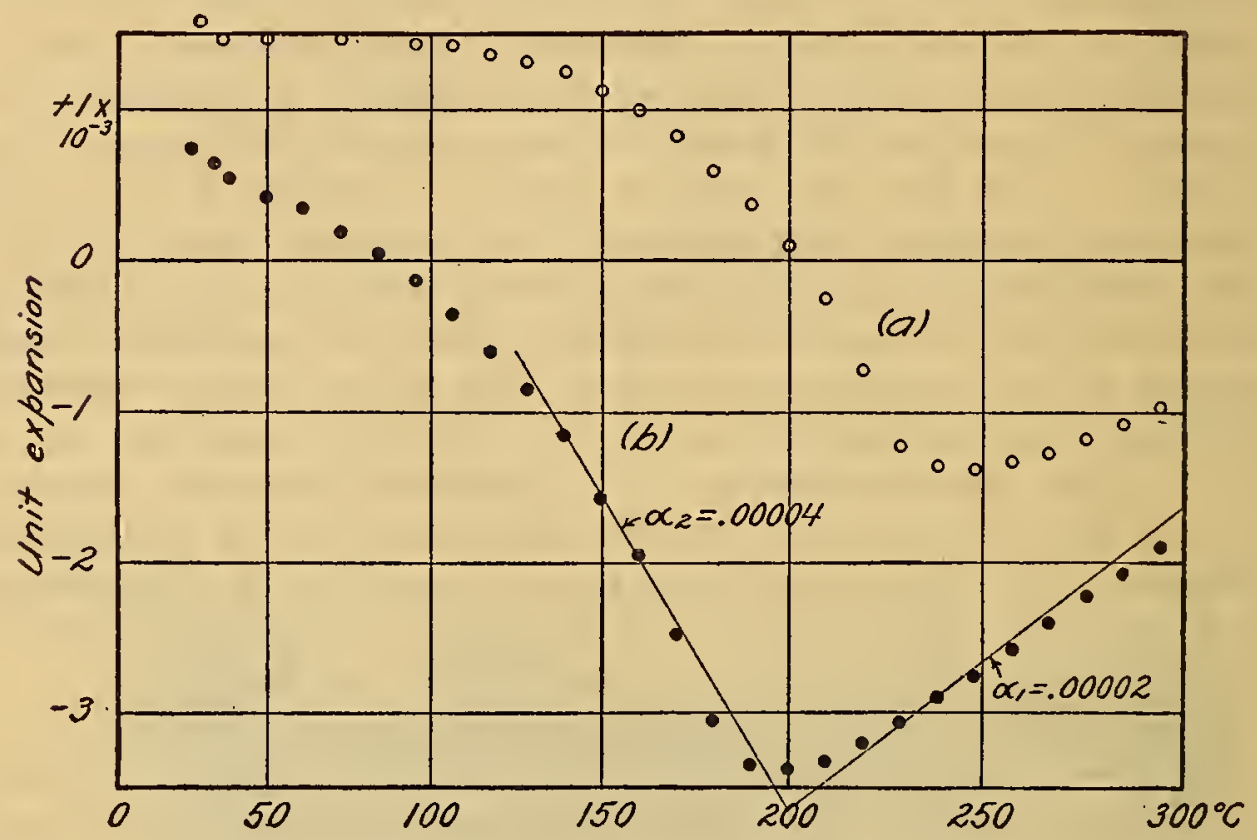

FIG. 10.-Unit length changes of $J$ composition cylinders 1 inch diameter and 4 inches long on cooling in an air stream after quenching in oil from $780^{\circ} \mathrm{C}$. for 15 seconds $(a)$, and from $850^{\circ} \mathrm{C}$. for 18 seconds $(b)$

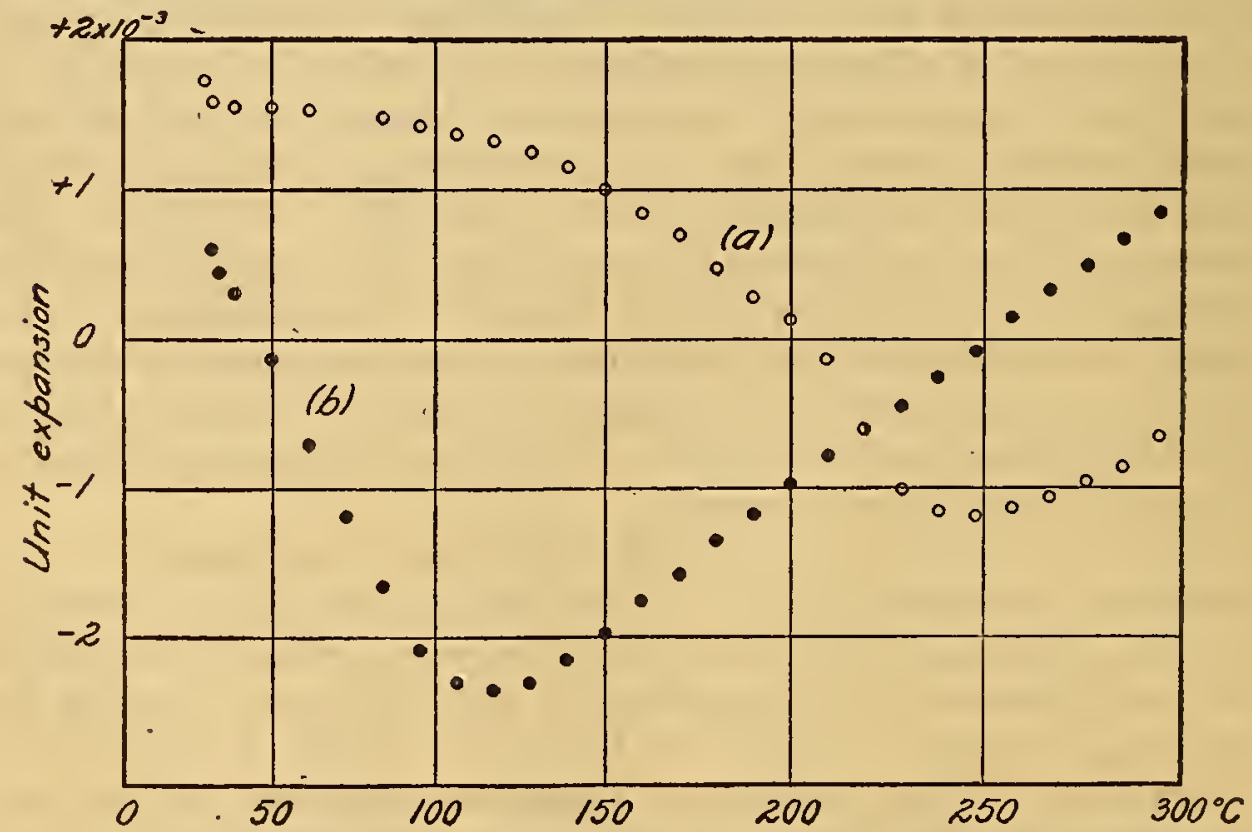

FIG. 11.-Unit length changes of standard $Z$ composition cylinders on cooling in an air stream after quenching in oil from $850^{\circ} \mathrm{C} .\left(\right.$ a), and from $1,000^{\circ} \mathrm{C} .(\mathrm{b})$, for 20 seconds 
the quenching temperature from $850^{\circ}$ C. to $1,000^{\circ}$ C. and scarcely complete at ordinary temperature.

Figure 12 gives the expansion curves for three compositions; two are structural alloy steels, curve $(a)$ and curve $(c)$, differing essentially only in carbon content, and the other is a high carbon-chromium alloy steel, curve $(b)$. Note the effect of about 0.10 per cent carbon in lowering $A r^{\prime \prime}$ about $130^{\circ} \mathrm{C}$, , (a) and (c). The transformation of

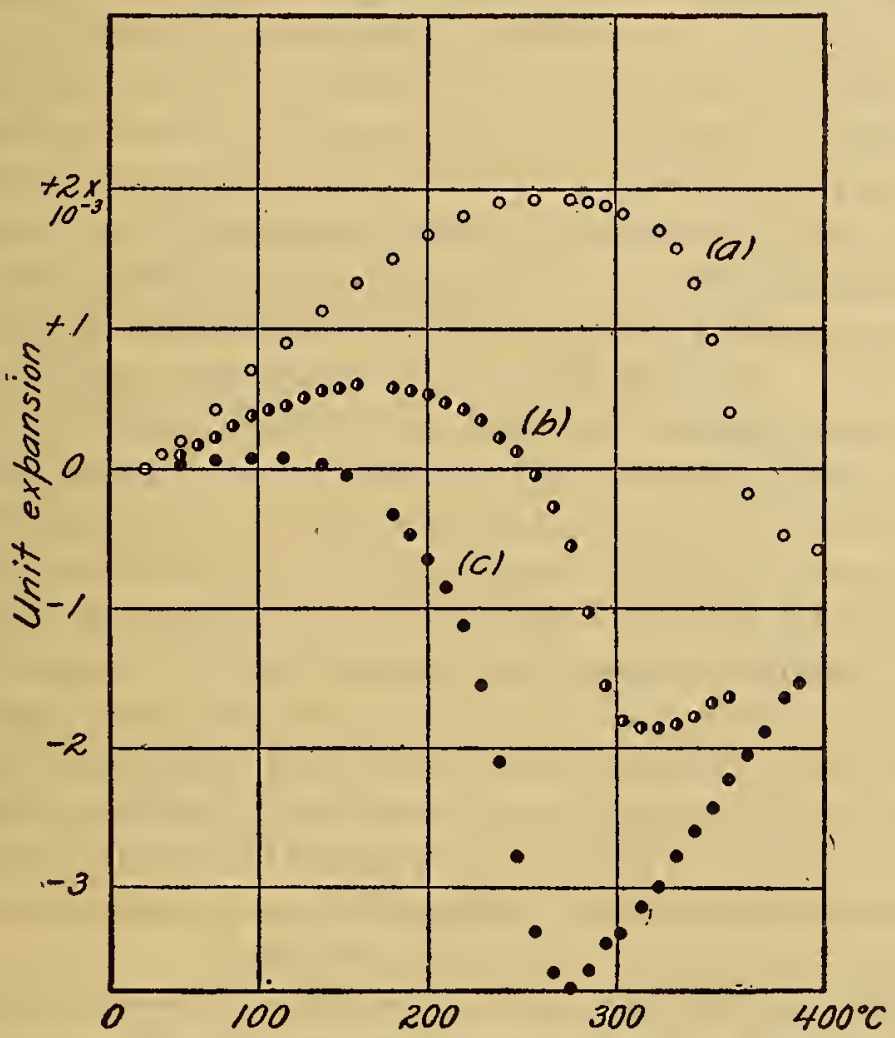

FiG. 12.-Unit length changes of standard cylinders on cooling in air stream after quenching in oil from $900^{\circ} \mathrm{C}$. for 10 seconds $(a)$ and $(c)$, and from $1,000^{\circ} \mathrm{C}$. for 20 seconds (b)

(a) is of composition $P,(b)$ of composition $Y$, and (c) of composition $Q$

the high alloy steel $Y$ comes between these two low-alloy steels and is quite similar in appearance. This is evidently due to the wellknown effect of chromium in reducing the solubility of carbon in iron.

It might be objected that these curves do not give the same expansion characteristics as would be found on continuous quenching. This is unquestionably true, but the difference is relatively small. The essential difference between continuous and interrupted quenching is in the time available for tempering of martensite as it forms. This transient tempering reduces somewhat the net length change on hardening, but the reduction is small in comparison with the total expansion and can safely be neglected for present purposes. On the other hand, if we should attempt to 
measure the expansion during fast cooling of convenient sizes the temperature differences between center and surface would distort the curve. The expedient adopted is therefore the lesser evil.

It is now evident that, through a considerable portion of the quenching range, the coefficient of expansion is negative; that is, the steel expands while cooling.

According to Table 3, permanent tensional stress can be developed at the surface during cooling only if the coefficient is negative and decisive evidence of such stress under certain quenching conditions is given. But the development of permanent tensional surface stress requires plastic deformation while the coefficient is negative; that is, while the steel is hardening. There can be no question of the ability of steel to deform plastically when austenitic, but its ability to deform plastically after $A r^{\prime \prime}$ starts might be questioned.

The transformation from austenite to martensite $A r^{\prime \prime}$ starts from isolated nuclei with the growth of martensite globules. In the initial stages the globules are small and independent, but as they grow contacts are made and a continuous network formed. So long as these globules are discontinuous they offer no effective barrier to plastic deformation of the remaining austenite, but once they join, the steel will be decidedly more elastic. There is a close analogy to this in the relation between hardness and carbon content of annealed carbon steels. With a carbon content of about 0.6 per cent, the pearlite network becomes continuous and causes an appreciable increase in the rate of hardness increase with carbon content. For an example of this see Figure 5 of a paper by Neville and Cain(14). It is therefore reasonable to assume that the proportional limit of steel remains low until $A r^{\prime \prime}$ is well advanced.

While the proportional limit is probably low during the initial stages of $A r^{\prime \prime}$, it is still not zero as assumed in the analysis of stress generation. The conception advanced will still hold, however, with the modification that the permanent stress will be determined by the deformation beyond that required to reach the proportional limit and hence will be lower than in the limiting case. Corrections for this departure from ideal conditions might be applied if we knew the elastic properties during $A r^{\prime \prime}$. The direct determination of these properties is evidently beyond the reach of the usual experimental methods, for the steel is not in a stable condition during $A r^{\prime \prime}$.

The elastic properties of the steel after the martensite network forms are also of importance in regard to the development of permanent stress. It has already been shown that the permanent stress develops as the temperature differences are reduced during this stage of quenching. Plastic deformation, which will modify the final permanent stress, can occur if the permanent stress developed at any stage is in excess of the proportional limit at that time. This situa- 
tion is, however, hardly probable, for the steel is continuously hardening as the permanent stress grows. Actually, it will be of consequence only if the cooling be completely stopped during the latter part of $A r^{\prime \prime}$ and then would have the effect of lowering the final permanent stress.

The temperature at which the steel becomes effectively elastic has been shown to be critically important in stress development. The above reasoning carried a little further leads to a method for its estimation. After the martensite forms a continuous network further transformation of the remaining austenite will produce pressure within these micro units. By the familiar theorem of Le Chatelier this pressure will lower the transformation temperature of the austenite. It follows therefrom that the transformation $A r^{\prime \prime}$ will progress approximately linearly until the martensite network is complete and continue thereafter at a rapidly decreasing rate. This is precisely the form that the expansion curves take.

The phenomenon of gradual linear expansion during $A r^{\prime \prime}$ is, of course, one of concentration change characteristic of the solidification of solid solution alloys and described by Rawdon and Epstein(15), page 385. They show, moreover, that some austenite is retained at ordinary temperatures in high-carbon steels. Such steel is therefore stressed within micro units as well as on the macro scale studied here. The existence of such stress is clearly revealed by the short cracks found in many specimens examined by Rawdon and Epstein.

By the above considerations alone the critical temperature at which steel becomes "effectively" elastic during quenching can be estimated from the expansion curves. It is evidently the temperature at which departure from linear expansion starts with falling temperature during $A r^{\prime \prime}$; that is, the analogue of the proportional limit of stressstrain curves. The chief characteristics of $A r^{\prime \prime}$ for the steels run, namely, the temperature of the start of $A r^{\prime \prime}\left(T_{\mathrm{m}}\right)$, and of the end of linear expansion $\left(T_{\mathrm{s}}\right)$, and the expansion from $T_{\mathrm{m}}$ to $T_{\mathrm{s}}, \alpha_{2}\left(T_{\mathrm{m}}-T_{\mathrm{s}}\right)$ are tabulated in Table 4 . As the maximum tensional surface stress is determined by the expansion from the minimum length to that at which the steel becomes elastic, $\alpha_{2}\left(T_{\mathrm{m}}-T_{8}\right)$ may be taken as a measure of it, or, in other words, of the susceptibility of the steel to cracking. The data given in the last column of this table are the temperature differences which will produce the total linear expansion, $\alpha_{2}\left(T_{\mathrm{m}}-T_{\mathrm{s}}\right)$, when $\alpha_{2}=0.4 \times 10^{-4}$ (see column 5) and also indicate the magnitude of the temperature drop that will produce maximum tensional stress. 
TABLE 4.-Expansion characteristics associated with the hardening transformation of some representative steels

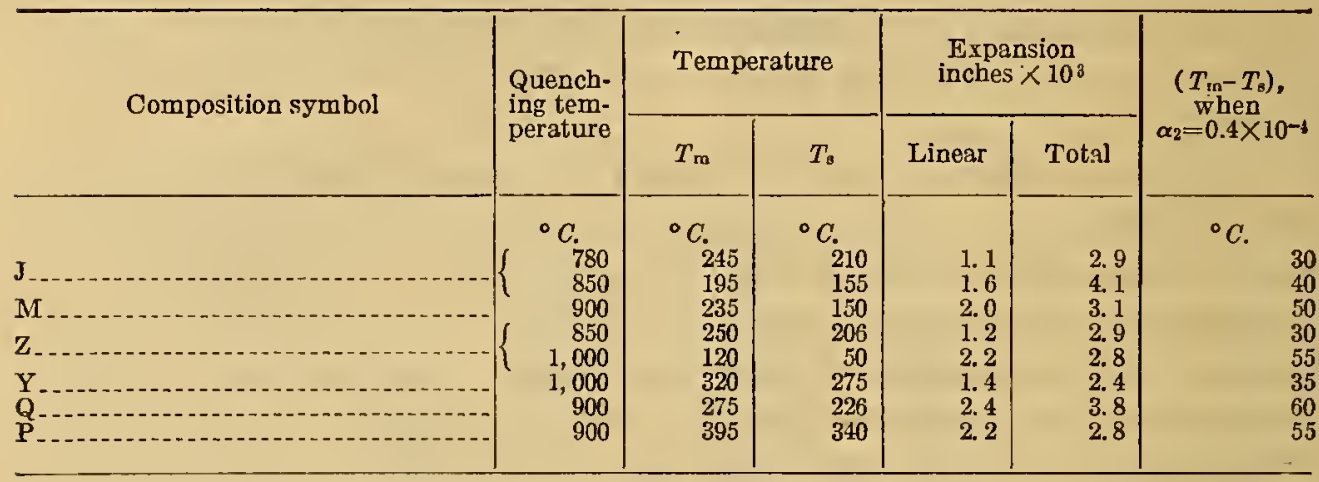

There are then two essential characteristics of the hardening transformation which determine the success of a particular quench that gives full hardening, namely, the linear expansion $\alpha_{2}\left(T_{\mathrm{m}}-T_{\mathrm{5}}\right)$ and the end of the range $T_{s}$. Both may be controlled by the composition and quenching temperature. They are, however, closely related for lowering $T_{s}$ increases the magnitude of $\alpha_{2}\left(T_{m}-T_{s}\right)$ in a particular steel so long as $A r^{\prime \prime}$ is completed above ordinary temperatures. Raising the quenching temperature of hypereutectoid steels lowers $T_{s}$ but increases $\alpha_{2}\left(T_{\mathrm{m}}-T_{\mathrm{s}}\right)$, which is favorable to cracking. There is, however, a thermal factor introduced by changing $T_{s}$ which opposes the above effect, namely, a decrease in the temperature difference with decreasing $T_{s}$. This factor will be investigated in the next section.

\section{TEMPERATURE DISTRIBUTION DURING QUENCHING}

It now remains to determine the temperature distribution in steel cylinders during quenching, in order that the induced stresses may be estimated for particular expansion characteristics of the steel. There are no less than five thermal variables which control the generation of stress in steel during quenching, namely, the initial temperature, the final temperature, the temperature at which the surface becomes elastic, the size of the cylinder, and the cooling power of the bath. To investigate the effect of all these variables experimentally is impracticable. Mathematical investigation of them is, however, feasible and yields simple general relations directly applicable to our problem. All that is required of experiment is the evaluation of two constants, one for the steel and one for the cooling medium. The applicability of mathematical analysis to problems of temperature distribution has already been indicated (11) and (16), so only the salient features will be developed here. 
Assume that the radial temperature gradient at surface points remote from the ends of a long cylinder is proportional to the surface temperature:

$$
\frac{d u}{d r}=-h u \text { when } r=c
$$

Here $u$ is the temperature on a ratio scale, $r$ the distance from the center, $c$ the radius, and $h$ a constant depending on the cooling medium and the steel; that is,

$$
h=\frac{H}{K}
$$

where $H$ is a property of the medium and $K$ the thermal conductivity of the steel. $K$ does not vary greatly under quenching conditions among the tool steels, so $h$ will be taken as a property of the bath alone, though its dependence on $K$ should not be ignored in other circumstances.

With this boundary condition and Fourier's conduction equation, the expression for the temperature at any point on a radius as a function of time can be derived by classical methods. This equation represents the cooling of steel in the common quenching media satisfactorily for present purposes. We need not introduce this cumbersome equation here; deductions from it are our chief concern.

The measure of the temperature gradient at the surface is alone insufficient to specify the temperature distribution, for it tells nothing about the effect of the radius $c$. The temperature gradient at the surface is independent of the radius. However, the temperature distribution from center to surface is a function of $h c$ and the time, or to use a more appropriate variable than time, the temperature at the surface. This complex relation can be expressed simply by curves for some particular temperature range and several values of $h c$ as in Figure 13. The temperatures are plotted against the square of the ratio $\frac{r}{c}$. This ratio is used because applicable to any size and it is squared for convenience in stress computations.

The ordinates on the left side of Figure 13 are the temperatures on the Centigrade scale. The relation of temperature to position is, however, somewhat more general than this manner of plotting would imply. To indicate this, the right-hand ordinates $u$ are used. Here the temperature is given on a ratio scale defined by:

$$
u=\frac{T_{\mathrm{r}}-T_{\mathrm{o}}}{T_{\mathrm{i}}-T_{\mathrm{o}}}
$$

where $T_{\mathrm{r}}$ is the temperature of a point $r$ distance from the center, $T_{\mathrm{i}}$ the initial temperature, and $T_{\mathrm{o}}$ the final temperature, the tem- 
peratures being taken on any scale. The temperature distributions given on the ratio scale, therefore, apply to any temperature range or scale so long as the surface has cooled 0.125 of the temperature range $\left(T_{\mathrm{i}}-T_{0}\right)$. For some other surface temperature ratio another set of curves would have to be computed. The curves will approach closer to a straight line, the smaller this ratio and depart farther therefrom the greater it is.

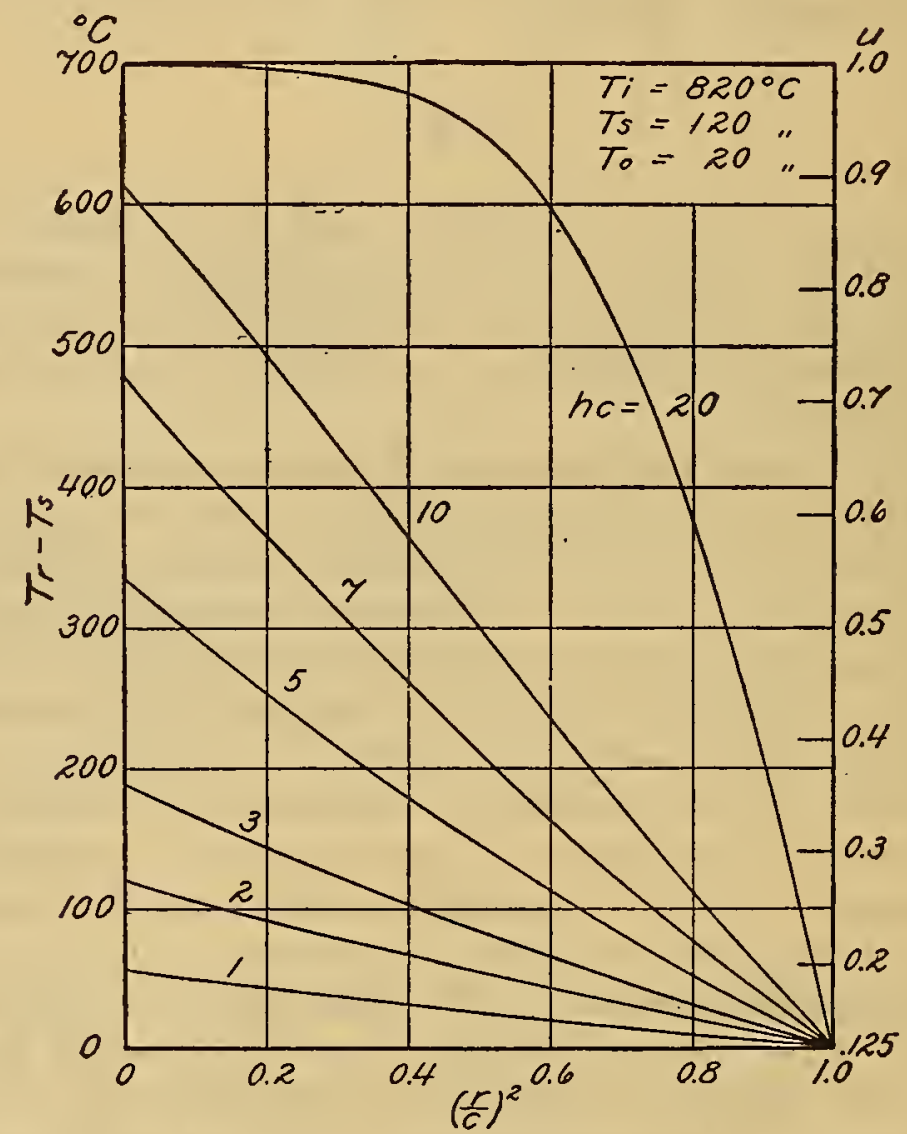

FIG. 13.-Temperature distribution from center, $\left(\frac{r}{c}\right)^{2}=0$, to surface, $\left(\frac{r}{c}\right)^{2}=1$, of a cylinder for several values of hc when the initial temperature, $T_{\mathrm{i}}$ is $820^{\circ} \mathrm{C}$.; the surface temperature, $T_{\mathrm{s}}$, is $120^{\circ} \mathrm{C}$.; and the final temperature, $T_{\mathrm{o}}$, is $20^{\circ} \mathrm{C}$.

We have now in $h c$ a variable which specifies the actual temperature distribution for particular initial, surface, and final temperatures. This permits the investigation of the relation between stress and $h c$ while the properties of the steel, the quenching temperature, and bath temperature remain constant. A more general variable which includes the latter thermal constants as well as $h c$ is, however, desirable. The temperature difference between center and surface, 
called for short the "temperature drop," supplies this need under certain restrictions.

If consideration be limited to low values of $h c$ or to times when the center has cooled through 0.3 or more of the temperature range, the temperature drop can be very simply expressed in terms of $h c$. Tensional surface stress is produced in tool steel only under just these conditions and is the chief cause of cracking, consequently this relation is well worth study in spite of its limitations.

The ratio of the surface temperature minus the final temperature $\left(T_{\mathrm{s}}-T_{\mathrm{o}}\right)$ to the center temperature minus the final temperature $\left(T_{\mathrm{c}}-T_{\mathrm{o}}\right)$ is a function of $h c$ as given in Figure 14 under the stated limitations.

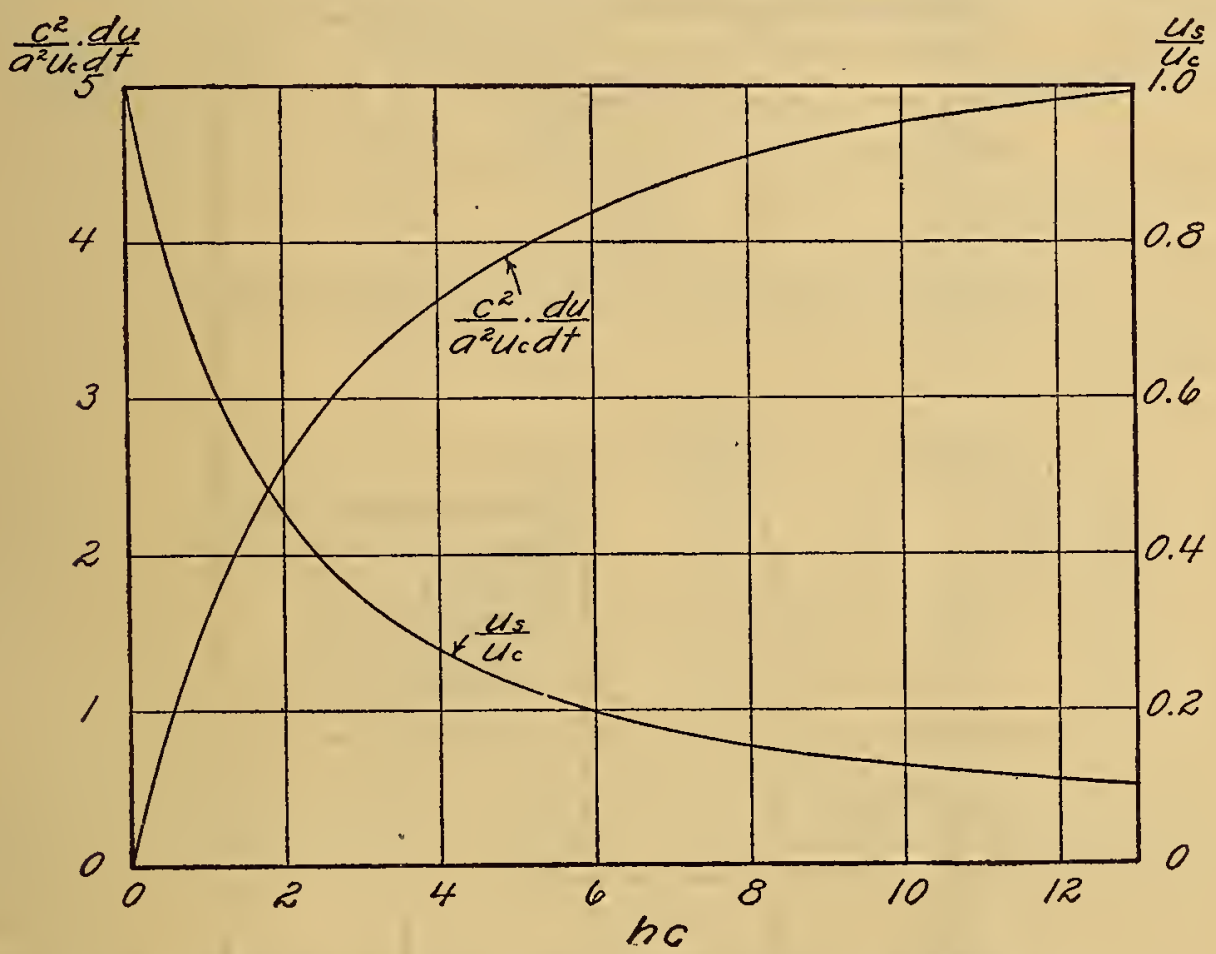

FIG. 14.-Relation between the ratio of the surface to the center temperature range, $\frac{T_{\mathrm{s}}-T_{\mathrm{o}} \text {, }}{T_{\mathrm{o}}-T_{\mathrm{o}}}$ and $h c$, curve (a). The cooling rate, $\frac{d u}{d t} t^{i m e s} \frac{c^{2}}{a^{2} u}$ is plotted against hc in curve $(b)$. These relations hold only when the center has cooled more than 0.3 of the temperature range or when hc is small

From this figure $\left(T_{\mathrm{c}}-T_{\mathrm{o}}\right)$ can be found for a given surface and final temperature, and by subtraction $\left(T_{\mathrm{c}}-T_{\mathrm{s}}\right)$. Note that the initial temperature does not enter, or, in other words, that the quenching temperature has no effect on the temperature drop under conditions which produce tensional surface stress. It is of some interest to note also the relation between the temperature drop and $h c$ for a specific temperature range as given in Figure 15.

The chief thermal effects from the stress viewpoint are those due to changes in the surface temperature $T_{\mathrm{s}}$ and in the final or bath 
temperature $T_{0}$. The effect of these variables is immediately evident on expressing the temperature drop in terms of $\left(T_{\mathrm{s}}-T_{\mathrm{o}}\right)$ and a function of $h c$ :

$$
\left(T_{\mathrm{c}}-T_{\mathrm{s}}\right)=\frac{(1-A)}{A} \cdot\left(T_{\mathrm{s}}-T_{\mathrm{o}}\right)
$$

where $A$ is the value of the ratio $\frac{\left(T_{s}-T_{o}\right)}{\left(T_{c}-T_{o}\right)}$ plotted in Figure 14 for a particular value of $h c$. For a given bath, then, $\left(T_{\mathrm{c}}-T_{\mathrm{B}}\right)$ is proportional to $\left(T_{\mathrm{s}}-T_{\mathrm{o}}\right)$. Hence, the higher $T_{\mathrm{s}}$ or the lower $T_{\mathrm{o}}$ the greater

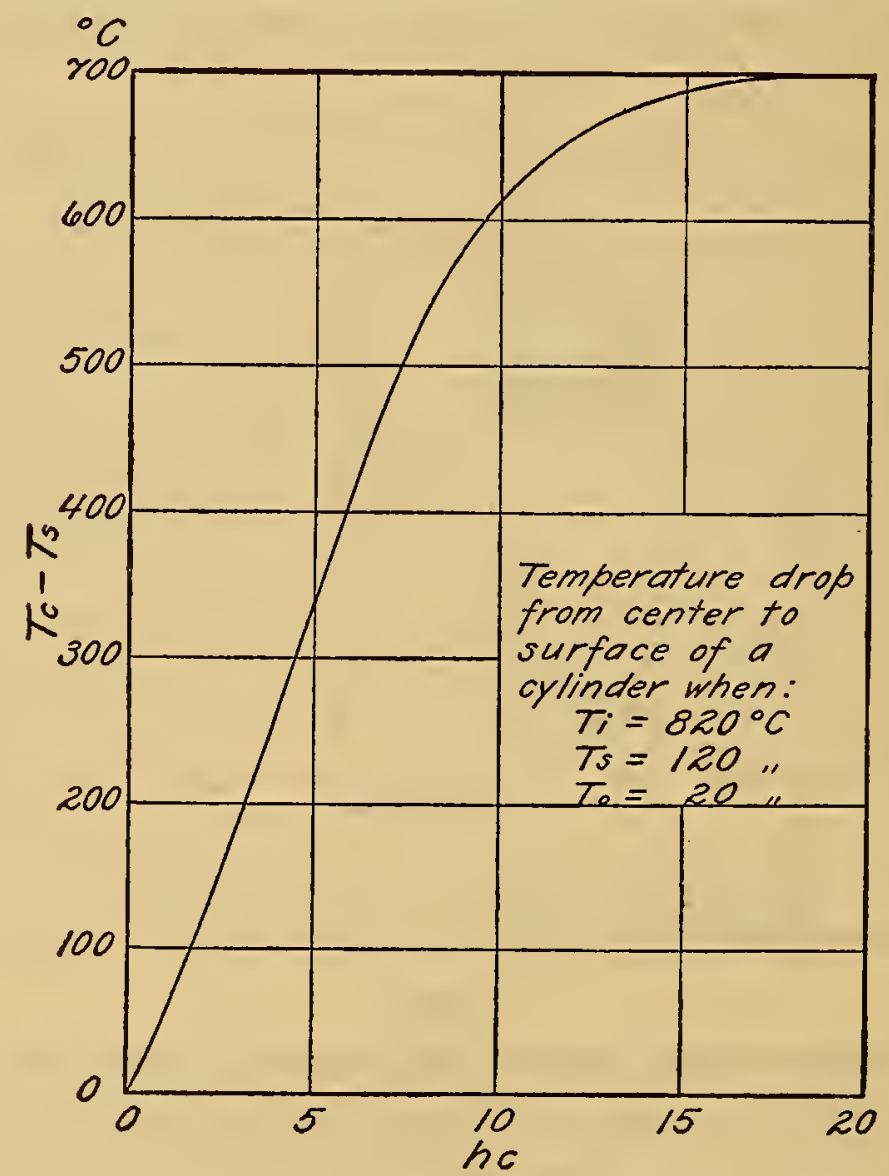

FIG. 15.-Relation between the temperature drop $\left(T_{\mathrm{o}}-T_{\mathrm{s}}\right)$ from center to surface of a cylinder and is the temperature gradient at the surface divided by the surface temperature and $c$ the radius in centimeters

the temperature drop. Thus $\left(T_{\mathrm{c}}-T_{\mathrm{s}}\right)$ as limited includes all the thermal factors determining the stress, so the relation between $\left(T_{\mathrm{o}}-T_{\mathrm{s}}\right)$ and the stress will be determined alone by the properties of the steel or, more specifically, by the characteristics of $A r^{\prime \prime}$

To apply these relations to practical conditions it is only necessary to evaluate the constant $h$ for the several quenching media used. 
This can be accomplished if the quenching curves for the center of a cylinder of known diffusivity are available: Figure 1 gives the required curves for a steel cylinder whose diffusivity has been estimated under quenching conditions by several devices. The relation between the cooling rate $\frac{d u}{d t}$, the diffusivity $a^{2}$, the radius $c$, the center temperature $u$ on the ratio scale, and $h c$ is given in Figure 14, but is limited to center temperatures less than 0.7 of the quenching range. From this relation values of $h$ were found for surface temperatures around $120^{\circ} \mathrm{C}$. as given in Table 5 and represent quenching with vertical stirring, about one cycle per second.

TABLE 5.-Estimated temperature drop $\left({ }^{\circ} \mathrm{C}\right.$.) from center to surface of solid cylinders during quenching in a prepared oil, neat's-foot oil, 60 per cent glycerin in water, and water when $T_{\mathrm{i}}=820^{\circ} \mathrm{C} ., T_{\mathrm{s}}=120^{\circ} \mathrm{C}$., $T_{\mathrm{o}}=20^{\circ} \mathrm{C}$.

\begin{tabular}{|c|c|c|c|c|c|}
\hline \multirow[b]{2}{*}{ Diameter in inches } & \multirow[b]{2}{*}{$\mathrm{C}$ radius } & \multicolumn{4}{|c|}{ Quenching medium } \\
\hline & & $\begin{array}{c}\text { Prepared } \\
\text { oil } \\
h=0.6\end{array}$ & $\begin{array}{l}\text { Neat's- } \\
\text { foot oil } \\
h=0.8\end{array}$ & $\begin{array}{l}60 \text { per } \\
\text { cent } \\
\text { glycerin } \\
h=3\end{array}$ & $\underset{\substack{\text { Water } \\
h=5}}{ }$ \\
\hline $\begin{array}{l}1 / 46 \\
3 / 8 \\
1 / 2 \\
3 / 4 \\
1\end{array}$ & $\begin{array}{r}C m . \\
0.32 \\
.48 \\
.63 \\
.95 \\
1.27\end{array}$ & $\begin{array}{l}10 \\
15 \\
19 \\
30 \\
41\end{array}$ & $\begin{array}{l}13 \\
20 \\
26 \\
41 \\
57\end{array}$ & $\begin{array}{r}53 \\
83 \\
110 \\
177 \\
248\end{array}$ & $\begin{array}{r}93 \\
147 \\
198 \\
317 \\
435\end{array}$ \\
\hline $13 / 2$ & $\begin{array}{l}1.90 \\
2.54 \\
3.81 \\
6.35\end{array}$ & $\begin{array}{r}64 \\
88 \\
140 \\
247\end{array}$ & $\begin{array}{r}88 \\
121 \\
192 \\
342\end{array}$ & $\begin{array}{l}385 \\
515 \\
647 \\
700\end{array}$ & $\begin{array}{l}598 \\
667 \\
700 \\
700\end{array}$ \\
\hline
\end{tabular}

The values of $h$ tabulated apply only to tool or high-alloy steels; that is, steels of the same diffusivity as the 32 per cent nickel steel. Such steels will cool at the same rate in the same media; $h$ varies considerably with the surface temperatures. In the case of oil, it may be twice as great for surface temperatures well above $120^{\circ} \mathrm{C}$. The rate of stirring has a pronounced effect on $h$ also. If oil is heated, $h$ remains fairly constant, but if water is heated, $h$ is greatly lowered at high-surface temperatures. These factors have important effects and must be carefully controlled to reproduce results.

The temperature relations will, of course, not be the same in a steel undergoing the $A r^{\prime \prime}$ transformation as in the transformationless nickel steel under otherwise identical conditions. However, the temperature distribution in the latter case can be taken as a reliable measure of the former, for the heat evolution of $A r^{\prime \prime}$ is a fairly constant quantity, depending as it does on the matrix, iron, and not on the variable solid solubility of carbide. In speaking of the temperature distribution in steel during hardening, it will be in this sense and will not imply that the true temperature distribution is known in any case. 
While $h$ has been evaluated for the cylinder alone, the values obtained apply to other simple shapes as well. The temperature drop is slightly higher in the sphere and lower in the slab than in the cylinder for the same value of $h c$, although the cooling rates will differ considerably among these shapes. Consequently, the stress relations to be developed are somewhat more general than might be implied from their development from the case of the cylinder alone.

\section{ESTIMATION OF PERMANENT STRESS}

The temporary axial stress, from which the permanent stress can be deduced by previous propositions, is given by:

$$
F_{\mathrm{a}}=\frac{C-\alpha\left(T_{\mathrm{r}}-T_{\mathrm{s}}\right)}{(e-f)}
$$

for points remote from the ends of a long cylinder; $e$ and $f$ are elastic constants and $\alpha$ the coefficient of expansion of the material, $T_{s}$ the temperature at the surface and $T_{\mathbf{r}}$ at some point on a radius at the instant in question, and $C$ a constant to be evaluated from the relation:

$$
\int_{0}^{\mathrm{c}} F_{\mathrm{a}} r d r=0
$$

To evaluate $C\left(T_{\mathrm{r}}-T_{\mathrm{s}}\right)$ must be expressed as a function of $r$, but from a preceding section $\alpha$ is a function of the temperature and must also be given in terms of the radius. The relation between $\alpha\left(T_{\mathrm{r}}-T_{\mathrm{s}}\right)$ and $r$ can be put in the form of a curve by combining a curve for $T$ v. $r$ (fig. 13) with a curve for $\alpha T$ v. $T$; that is, an expansion curve, but it can not be put into the form of an easily integrable equation. However, $C$ can be evaluated without integration by a simple graphical method.

If $\alpha\left(T_{\mathrm{r}}-T_{\mathrm{s}}\right)$ be plotted against $\left(\frac{r}{c}\right)^{2}$ for a particular temperature distribution, the deformation origin must be so chosen that the positive and negative areas between it and the curve are equal in order to satisfy the second equation. This gives $(e-f) F_{a}$ directly, but leads to no very general relations. If, however, we make the simplifying assumption that the temperature varies linearly with the square of the radius, as it does on linear cooling and approximates on exponential cooling when the temperature drop is small, then a general equation for the temporary stress can be easily derived. This assumption introduces no great error when confined to the case of tensional surface stress which is the most important and general one.

Assume, further, that the contraction before $A r^{\prime \prime}$ is linear and given by $\alpha_{1}$ to the temperature of minimum length $T_{m}$; that the 
expansion from $T_{m}$ to the temperature at which the steel becomes effectively elastic $T_{\mathrm{s}}$ is also linear and given by $\alpha_{2}$. These assumptions represent actual expansion with complete hardening sufficiently close for practical purposes (see fig. 10). The variables $\alpha_{1}, \alpha_{2}, T_{\mathrm{m}}$, and $T_{\mathrm{s}}$ are properties of the steel. One other variable is needed to completely define the stress situation, namely, the center temperature, when the surface is at $T_{\mathrm{s}}, T_{\mathrm{c}}$, or $\left(T_{\mathrm{c}}-T_{\mathrm{s}}\right)$. This latter variable is a thermal property as already defined. What is desired now is to find a general relation between the temperature drop $\left(T_{\mathrm{c}}-T_{\mathrm{s}}\right)$ and the stress as determined by $\alpha_{1}, \alpha_{2}, T_{\mathrm{m}}$ and $T_{\mathrm{s}}$. By simple analytical considerations the constant $C$ can be found as indicated above:

$$
C=\frac{\alpha_{1}\left(T_{\mathrm{c}}-T_{\mathrm{m}}\right)^{2}-\alpha_{2}\left(T_{\mathrm{m}}-T_{\mathrm{s}}\right)^{2}}{2\left(T_{\mathrm{c}}-T_{\mathrm{s}}\right)}
$$

from which the axial surface stress can be written:

$$
(e-f) F_{\mathrm{a}}=\frac{-\left(T_{\mathrm{c}}-T_{\mathrm{s}}\right)}{2}\left[\alpha_{2}+\left(\alpha_{1}-\alpha_{2}\right)\left(\frac{T_{\mathrm{m}}-T_{\mathrm{g}}}{T_{\mathrm{c}}-T_{\mathrm{g}}}-1\right)^{2}\right]
$$

From this expression the stress can easily be computed for values of $\left(T_{\mathrm{m}}-T_{\mathrm{s}}\right),\left(T_{\mathrm{c}}-T_{\mathrm{s}}\right)$ variable, as in Figure 16. The values of $\alpha_{1}=$ 0.000020 and $\alpha_{2}=-0.000040$ correspond closely with the conditions of Figure $10(b)$, and hence with the conditions of the experiments on quenching cracks. Only positive values, tensional stress, are computed, for the expression does not hold for large temperature drops, a case which is considered later, although it is quite evident that the surface stress goes over to compression for high temperature drops.

This fundamental equation states precisely what the quenching experiments showed qualitatively. It shows that as the temperature drop is increased from zero the stress rapidly increases in tension to a maximum, then decreases, passing through zero into compression. From the viewpoint of quenching cracks, the magnitude of the maximum and the range of drops over which tensional stress is obtained are highly important characteristics of the steel. The condition for maximaum tensional stress is easily found by differentiating the expression and equating to zero, whence:

$$
\frac{\left(T_{\mathrm{c}}-T_{\mathrm{s}}\right)^{2}}{\left(T_{\mathrm{m}}-T_{\mathrm{s}}\right)^{2}}=\frac{\alpha_{1}-\alpha_{2}}{\alpha_{1}}
$$

For the particular values of $\alpha_{1}$ and $\alpha_{2}$ chosen, the stress is a maximum when:

$$
\frac{\left(T_{\mathrm{c}}-T_{\mathrm{s}}\right)}{\left(T_{\mathrm{m}}-T_{\mathrm{s}}\right)}=\sqrt{3}
$$

Substituting this ratio in the original equation, it is evident that the magnitude of the maximum stress is directly proportional to $\left(T_{\mathrm{m}}-T_{\mathrm{s}}\right)$, $\alpha_{1}$ and $\alpha_{2}$ constant. 
The range of drops producing tensional stress is found by putting the original equation for stress equal to zero:

$$
\frac{\left(T_{\mathrm{m}}-T_{\mathrm{s}}\right)}{\left(T_{\mathrm{c}}-T_{\mathrm{s}}\right)}=1-\sqrt{\frac{-\alpha_{2}}{\alpha_{1}-\alpha_{2}}}
$$

and for the above values of $\alpha_{1}$ and $\alpha_{2}$ :

$$
\left(T_{\mathrm{c}}-T_{\mathrm{s}}\right)=5.45\left(T_{\mathrm{m}}-T_{\mathrm{s}}\right)
$$

Thus, the range of temperature drops over which tne stress at the surface is tensional is directly proportional to $\left(T_{\mathrm{m}}-T_{\mathrm{s}}\right)$. These last two conclusions are, of course, evident from the plotted curves of Figure 16. From them it follows that the magnitude of the tensional

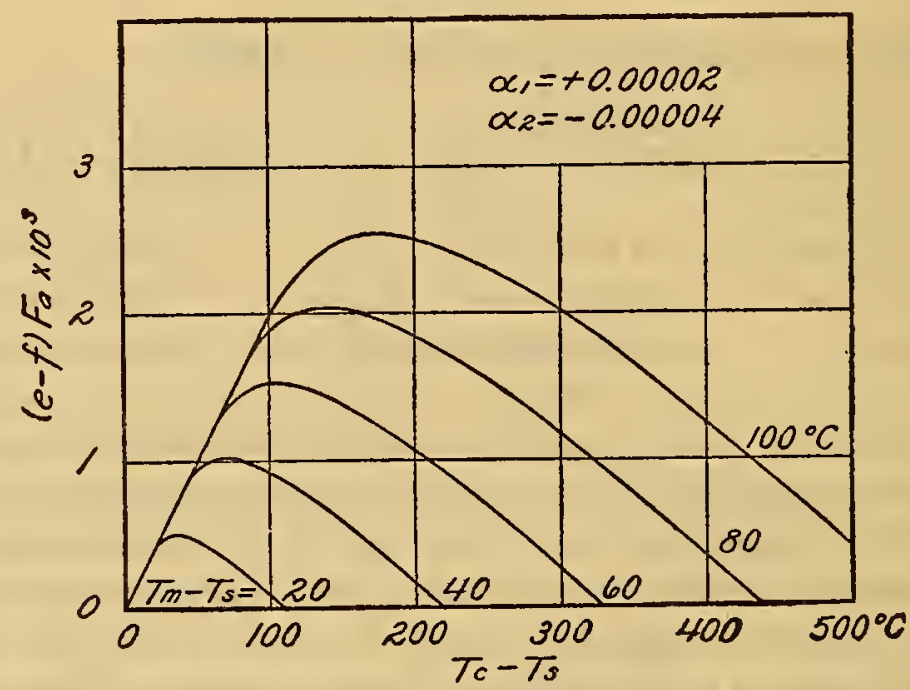

FIG. 16.-Computed tensional stress at the surface of long cylinders as a function of the temperature drop for several values of $\left(T_{\mathrm{m}}-T_{\mathrm{s}}\right)$, the temperature range of linear expansion during $A r^{\prime \prime}$

surface stress, and hence the danger of cracking, will be greater the larger $\left(T_{\mathrm{m}}-T_{\mathrm{s}}\right)$ and that dangerous stress will be encountered over a greater range of sizes and cooling powers, the larger $\left(T_{\mathrm{m}}-T_{\mathrm{s}}\right)$.

Before proceeding further with the interpretation of our stress equation it will be well to inquire whether or not it represents faithfully the results of experiment. It certainly corresponds with the observation of Section II that quenching cracks due to tensional surface stress occur only when the temperature drop is fairly small and not when the drop is very small or very large. Compressional surface stress was observed experimentally when the drop was large (Fig. 2), as required by the equation. A quantitative comparison is, however, quite difficult, for, in the region covered by the equation, the steel usually cracks, thereby preventing stress measurements by 
the Heyn method. But if the crack is single and deep, its width at the surface may be taken as a crude measure of the stress there. The width of such cracks is given in Table 6 . There is a consistent increase in the width, independent of the diameter or quenching medium, with increasing temperature drop from 30 to $200^{\circ} \mathrm{C}$. Cracks abruptly disappear at higher drops. The width of the crack, however, does not afford a reliable criterion of the maximum surface tensional stress, for the stress distribution becomes more complicated as the maximum is approached and passed. It is quite possible that with a surface stress lower than the maximum the crack will open wider than at the maximum if the drop is higher than at the maximum.

The location of the maximum tensional surface stress in terms of the temperature drop is an important problem. It, of course, depends on the value of $\alpha_{2}\left(T_{\mathrm{m}}-T_{\mathrm{s}}\right)$. From analytical considerations $\left(T_{\mathrm{m}}-T_{\mathrm{g}}\right)$ is $40^{\circ} \mathrm{C}$. and $\alpha_{2}=-0.00004$, Table 4 , for the steel and quenching temperature used. $\left(T_{\mathrm{m}}-T_{\mathrm{s}}\right)=60^{\circ} \mathrm{C}$. has been arbitrarily selected for further computations. This value gives compressional surface stress for water quenching a 1-inch cylinder as observed so is probably of the right magnitude, although it probably exaggerates the tensional stress range.

TABLE 6.-Width of crack at surface of specimens which cracked as quenched in the media noted. All specimens of $J$ composition quenched from $850^{\circ} \mathrm{C}$.

\begin{tabular}{|c|c|c|c|c|}
\hline Diameter in inches & Quenching medium & $h c$ & $\begin{array}{c}\text { Estimated } \\
\text { temperature } \\
\text { drop }\end{array}$ & Width of crack \\
\hline & $\begin{array}{l}\text { Prepared oil } \\
\text { Neat's-foot oil } \\
60 \text { per cent glycerin } \\
\text { Neat's-foot oil }\end{array}$ & $\begin{array}{l}0.57 \\
.76 \\
1.4 \\
1.5\end{array}$ & $\begin{array}{ll}{ }^{\circ} \mathrm{C} & \\
& 30 \\
41 \\
\\
83 \\
\\
88\end{array}$ & $\begin{array}{r}m m \\
0.028 \\
.028 \\
.031 \\
.042\end{array}$ \\
\hline & $\begin{array}{l}\text { Water } \\
60 \text { per cent glycerim } \\
\text { Water- }\end{array}$ & $\begin{array}{l}1.6 \\
1.9 \\
2.4 \\
3.2\end{array}$ & $\begin{array}{r}93 \\
110 \\
147 \\
198\end{array}$ & $\begin{array}{l}.035 \\
.045 \\
.052 \\
.080\end{array}$ \\
\hline
\end{tabular}

Unfortunately, the writer has not been able to determine the value of this characteristic of the steel by any other method. It is believed, however, that a qualitative correspondence has been established between the results of experiment and the analytical results. This indicates that the fundamental conceptions are correct. It is therefore well to see what further information can be gained from the stress equation. Any new conclusion drawn from it which can be directly tested by experiment is, of course, a further test of its validity, and certain ones will be noted.

The expansion from the temperature at which the expansion curve is a minimum (start of $A r^{\prime \prime}$ ) and the temperature at which the steel becomes effectively elastic, symbolically $\alpha_{2}\left(T_{m}-T_{\mathrm{s}}\right)$, is found 
to be directly responsible for the deep-seated cracks produced by surface tension. If this expansion is zero, the permanent stress at the surface can only be compressional, which is the case with an austenitic steel. Evidently the magnitude of the permanent tensional stress at the surface can be altered by means of $\alpha_{2}\left(T_{\mathrm{m}}-T_{\mathrm{s}}\right)$. The expansion curves and Table 4 show that the magnitude of this property of the steel can be controlled by the composition and quenching temperature. Much may be accomplished by adjustment of the composition, but the present problem is how to control the stresses in the available compositions. Analysis and experiment show that a low quenching temperature reduces the liability to crack, but it may be questioned that this is due entirely to the lower tensional stress attained thereby. Steel is probably inherently

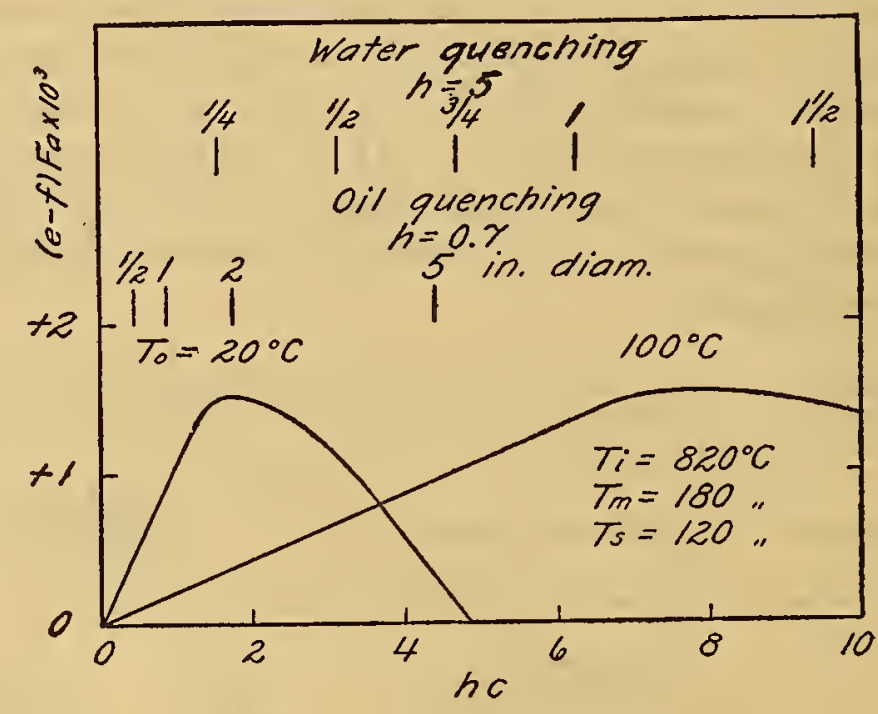

FIg. 17.-Effect of the bath temperature, $T_{\mathrm{o}}$, on the surface stress. The diameters of cylinders which will give the stresses indicated are estimated on the assumption that the cooling power of the bath, $h$, does not change with the bath temperature

more brittle as quenched from a high temperature than from a low one because of the coarser microstructure. Although lowering the quenching temperature is an effective means of aroiding cracks, the problem stated at the outset is how to quench safely from a high temperature. We must, therefore, turn to the thermal variables for practical expedients to control stress generation.

The curves of Figure 16 show that the major cause of quenching cracks, high tensional surface stress, may be avoided by cooling in such a manner that the temperature drop $\left(T_{\mathrm{c}}-T_{\mathrm{s}}\right)$ is either very small or very large when the surface becomes elastic. For reasons to be considered later, low surface stress obtained by a small drop is generally preferable to the same obtained with a large drop. A 
small drop can, of course, be obtained in a particular size by using a very slow quenching medium but introduces the difficulty of producing full hardness. The expression for the temperature drop in terms of $\left(T_{c}-T_{0}\right)$ given in Section $V$ shows how a low drop can be obtained at low temperatures with a fast quenching medium. It states that for a given value of $h c$ the drop is directly proportional to $\left(T_{5}-T_{\circ}\right)$. Thus, the drop can be lowered by raising $T_{\mathrm{o}}$ or reducing $T_{\mathrm{s}}$; that is, by raising the bath temperature or by lowèring $A r^{\prime \prime}$. This is illustrated by Figure 17. Here the surface stress is plotted against $h c$

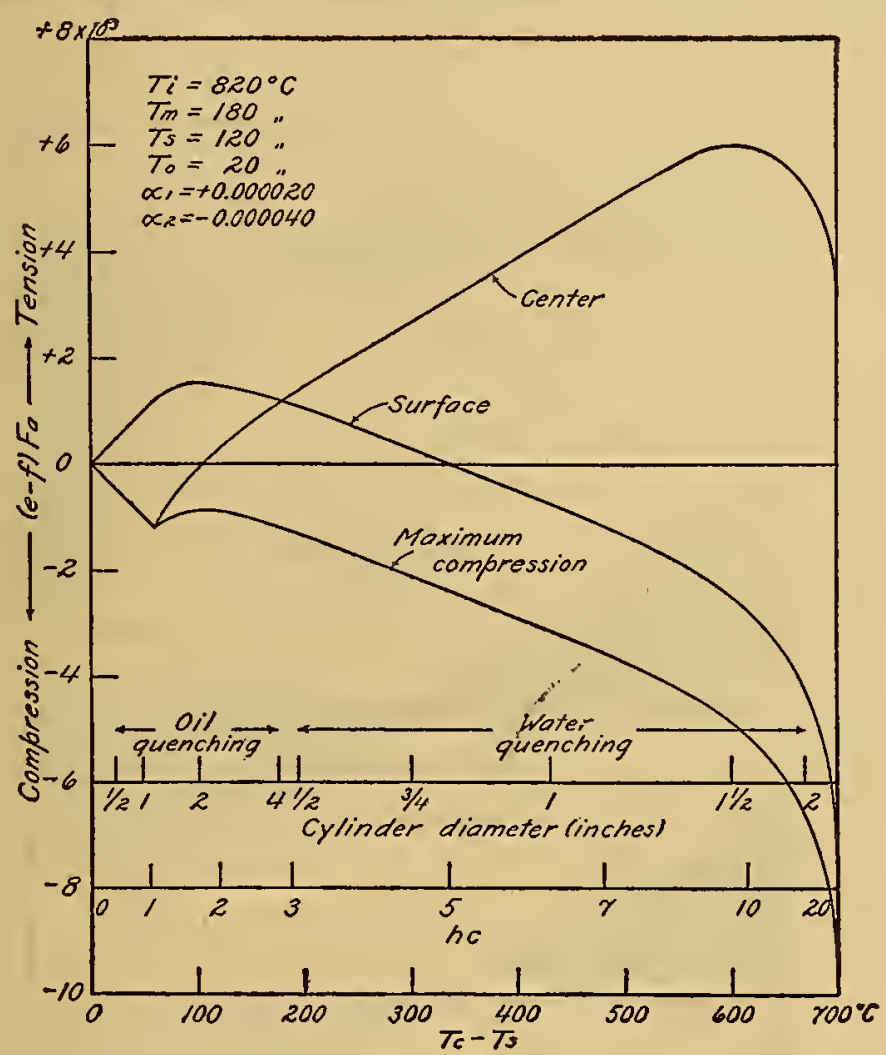

FIG. 18.-The computed axial stress at the center and surface and the maximum compressional stress as a function of the temperature drop $\left(T_{\mathrm{o}}-T_{\mathrm{s}}\right)$, within a steel cylinder quenched from 820 to $20^{\circ} \mathrm{C}$. when the surface is at $120^{\circ} \mathrm{C}$.

It is assumed that the coefficient of expansion of the steel as austenite is +0.00002 and during the first $60^{\circ} \mathrm{C}$. of $A r^{\prime \prime}$ is -0.00004 . Values of $h c$ and the diameters of cylinders for oil and water quenched which will give certain temperature drops are indicated

for surface temperatures of $20^{\circ}$ and $100^{\circ} \mathrm{C}$., other things the same. Though generality is lost by so plotting, the results are given in terms of quenching media and sizes, the variables of practice. This figure implies that $h$ is constant with changing bath temperature, which is far from true in the case of water. 
So far only the axial stress at the surface produced by small temperature drops has been considered. The stresses within the cylinder and those produced by large temperature drops are also of some importance. The axial stress at the center produced by low temperature drops is found in the same manner as that at the surface and is given by

$$
(e-f) F_{\mathrm{a}}=\frac{-\left(T_{\mathrm{c}}-T_{\mathrm{s}}\right)}{2}\left[\alpha_{1}-2 \alpha_{1} \frac{\left(T_{\mathrm{m}}-T_{\mathrm{s}}\right)}{\left(T_{\mathrm{c}}-T_{\mathrm{s}}\right)}+\left(\alpha_{1}-\alpha_{2}\right) \frac{\left(T_{\mathrm{m}}-T_{\mathrm{s}}\right)^{2}}{\left(T_{\mathrm{c}}-T_{\mathrm{s}}\right)^{2}}\right]
$$

For comparison with the surface stress it has been computed and plotted with the surface stress in Figure 18.

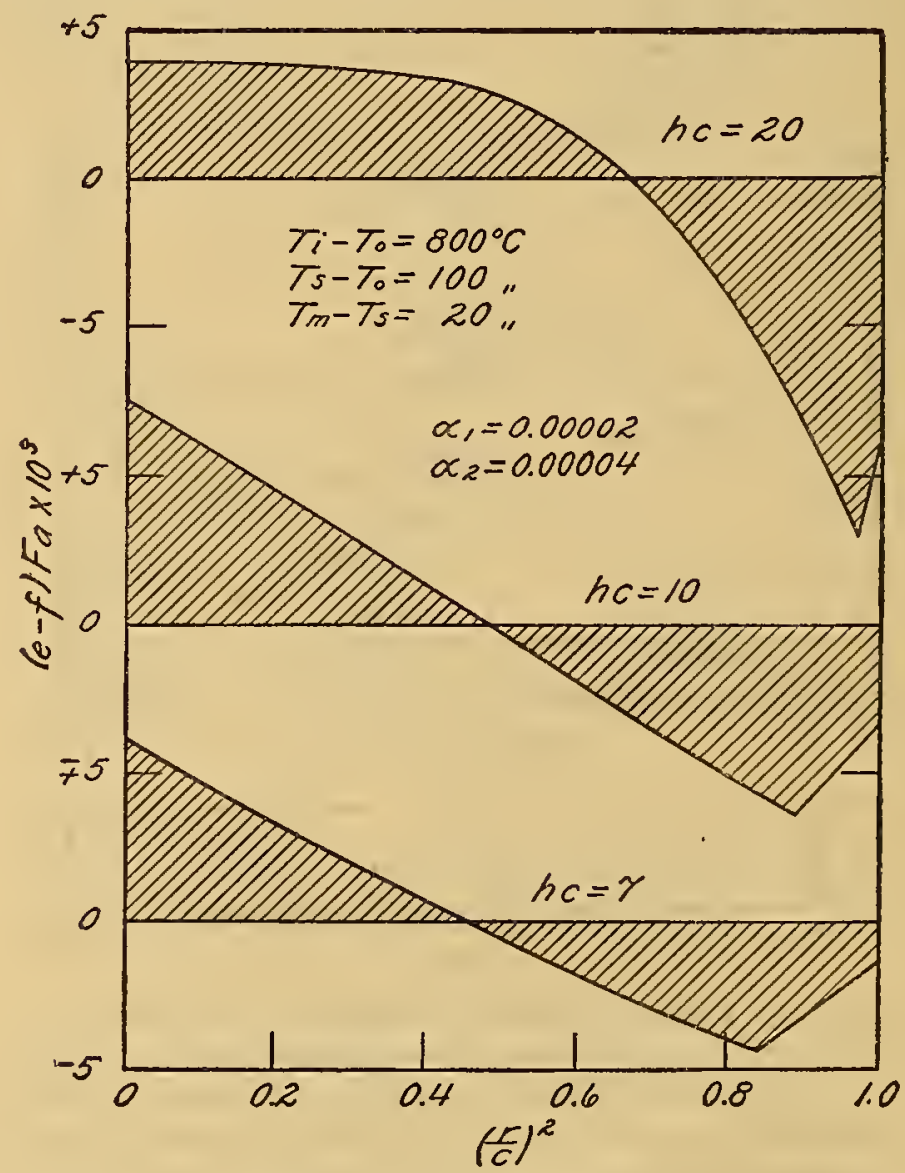

Frg. 19.-Computed axial stress distribution within cylinders for high values of hc

Only the portions of the curves of Figure 18 corresponding to low values of $\left(T_{\mathrm{c}}-T_{\mathrm{s}}\right)$ were obtained from the above equations. With large values of $\left(T_{\mathrm{c}}-T_{\mathrm{B}}\right)$ the temperature versus $\left(\frac{r}{c}\right)^{2}$ curves are no longer even approximately linear (fig. 13), as assumed in the derivation of these equations, so the method eraluating $C$ first mentioned was used. Figure 19 illustrates the method. The values of $\alpha_{1}, \alpha_{2}$, and 
$\left(T_{\mathrm{m}}-T_{\mathrm{s}}\right)$ given in Figure 18 are used but with the temperature distributions given by Figure 13. The deformation versus $\left(\frac{r}{c}\right)^{2}$ curves of Figure 19 were first plotted from an arbitrary deformation origin and the origin then shifted so that the positive and negative shaded areas were equal. In this way the curves of Figure 18 were extended to high values of $\left(T_{\mathrm{c}}-T_{\mathrm{s}}\right)$.

Figure 19 shows that the compressional stress is not a maximum at the center for large drops and that the maximum approaches closer to the surface the greater the drop, or $h c$, but never reaches it. It was observed experimentally that the maximum compressional stress is below the surface when the surface is under compression (fig. 2). In fact, the difference between the surface and maximum stress under these conditions gives a measure of $\alpha_{2}\left(T_{\mathrm{m}}-T_{\mathrm{s}}\right)$. The value of the maximum compressional stress is that of $C$, and it is also plotted in Figure 18. The maximum and the surface stresses for large drops increase continuously with the drop, but the center stress has a maximum in tension for a drop of about $600^{\circ} \mathrm{C}$.

This figure gives only the axial stresses. Cracks are usually due to tangential stress, but the axial stress is more easily computed and is directly comparable with stress measurements by the Heyn method. At the surface the tangential stress is equal to the axial, but at the center it is half the axial stress. Interior transverse cracks might then be anticipated when the surface is under high compression. The writer has heard of such cracks but has never seen any.

Figure 18 shows further that the tensional surface stress, the chief cause of cracks, is relatively low in comparison with the other stresses. In fact, a very high tensional stress can not be produced at the surface because it is restricted by $\alpha_{2}\left(T_{\mathrm{m}}-T_{\mathrm{s}}\right)$. This means that the steel must crack under relatively low and apparently uniform surface stress. The reasons for the failure of a material of such high elastic properties as tool steel under low loads are, however, quite obvious. While the stress may appear to be uniformly distributed, there are actually stress concentrations due to mechanical discontinuities in the surface and even within the steel. It is well known that local stress is greatly magnified by such effects. Often the cracks start in the slight depressions produced by stamping identifying numbers on the specimens. The stress concentration follows the bottom of the crack and thereby extends it. Another factor contributing to the development of cracks is the microstress produced by the austenite retained under pressure as explained (p. 419).

It may be doubted that cracks as found by Rawdon and Epstein form with a normal quenching temperature, but high microstresses are nevertheless present and require little additional stress to develop microcracks which are then extended by the resulting stress concentrations. 
Having developed the relations between stress and the quenching treatment of steel, we may now profitably consider methods for the control of stress and the elimination of quenching cracks.

\section{CONTROL OF QUENCHING CRACKS}

In the experimental study of quenching cracks two types were recognized, namely, local cracks associated with sharp changes in contour and deep-seated cracks which follow the major axes of the piece. Only the latter type was investigated. It was found to be caused chiefly by tensional stress at the surface. Incidental observations showed that shapes having local cracks may or may not contain deep-seated cracks. Local cracks may occur when the surface is under high compression or tension. Hence there is more latitude for the control of stress in shapes not subject to local cracking than otherwise. It is therefore useful to classify shapes submitted for hardening according to whether or not they are susceptible to local cracking, or briefly as complicated or simple shapes.

Among the complicated shapes are also included simple shapes having interior cavities not effectively cooled by the quenching medium, as, for example, $(a)$ and $(b)$ of Figure 4. This distinction is important, for a treatment which will successfully harden the same shape when solid may crack it when an interior cavity is present. The reason for this is that the interior can deform plastically after the surface hardens. The stress will then be determined by the temperature distribution when the interior section becomes elastic.

Another important classification from the viewpoint of stress is compositions which harden completely as treated and those which harden only to a moderate depth, here styled surface-hardening steels. The preceding analysis of stress generation has been confined to steels which harden completely with but casual mention of the surface-hardening steels. The latter class were shown to be under high compressional stress at the surface as quenched, due chiefly to the constitutional difference between the center and surface regions. Carbon tool steel is the most common representative of this class. However, for sizes under about one-half inch diameter with water quenching it must be considered as a deep-hardening steel also, for it then hardens completely. Water quenching simple shapes which harden on the surface only introduce no difficulty with cracking. In complicated shapes, however, difficulty is likely to be experienced with distortion and local cracking when water quenching is required, as is almost always the case with surface-hardening steels. Also more desirable physical properties for some purposes are obtained with full hardening.

The mechanical properties attendant on full hardening can be obtained in simple shapes with economy of alloy content by water 
quenching. Familiar examples of this are simple structural alloy steel shapes and ball bearings, the compositions of which are adjusted to the size so as to give full hardening on water quenching. The high compressional stress at the surface of large sizes so treated is no handicap, for it is fully relieved by tempering in the case of structural steels, and residual surface compression may even be an advantage in the case of ball bearings. With complicated shapes, however, recourse must be taken to slower quenching media in order to avoid distortion and local cracking.

In this connection it might appear desirable to use intermediate quenching rates between water and oil. In general, this is not desirable. The stress curves of Figure 18 and the experimental results show that either a fast or a very slow quench will give low surface stress. However, to obtain the low surface stress with a fast cooling rate would require a quenching bath for every size. Moreover, the stress is not zero below the surface and, in complicated shapes, would probably cause cracks. Consequently, it is preferable to aim for high compression; that is, use water quenching if the shape permits, or return to the problem of oil quenching.

Oil quenching many common sizes and types of deep-hardening steel introduces considerable difficulty with deep-seated cracks. Quenching experiments with a particular oil-hardening composition showed that cylinders $1 / 2$ inch diameter and under are not seriously stressed by oil quenching nor are sizes over $1 \frac{1}{2}$ inches which do not harden completely. The difficulty with deep-seated cracks is therefore restricted, in this particular case, to sizes from about $3 / 4$ inch to $11 / 2$ inches diameter. This is, of course, an important range of sizes.

The freedom from deep-seated cracks obtained when the center of large sizes does not harden on oil quenching suggests that this factor might be introduced in smaller sizes by appropriate modification af the composition. If, however, the alloy content be so reduced that cylinders under about 2 inches diameter do not harden to the center in oil, it will then be difficult to obtain full and uniform surface hardening on oil quenching. This situation is well illustrated by chromium-bearing steel, composition $M$, which will seldom harden completely on oil quenching 1 -inch diameter cylinders. When it does not harden completely, the soft constituent, troostite, is nearly as prevalent at the surface as at the center. The reason for this is, of course, that, with the small size and slow cooling, there is no great difference in cooling rate between the center and surface at the temperature of $A r^{\prime}$. It therefore follows that for moderate sizes of complicated shapes a steel which hardens completely on oil quenching is required.

This reduces the problem to that of avoiding quenching cracks on oil quenching deep-hardening steels in moderate sizes. In practice, 
deep-seated cracks of visual size are probably not so common as might be inferred from the results given here. This is due to the normal practice of using a lower quenching temperature and of tempering immediately after quenching. Prompt tempering probably reduces the chances of a crack developing to a visual size but may not inhibit the formation of microscopic cracks. The fact that cracks generally develop slowly, as noted in Section II, makes it highly probable that they are preceded by microscopic cracks which form as soon as ordinary temperatures are reached, or even before. Prompt tempering may inhibit the growth of such cracks but still leave a nucleus from which failure may start in service. Hence, this expedient can not be considered a satisfactory solution of the problem.

The subcommittee on tool steel of the A. S. S. T. (18) reoommends a more bold treatment for steel of the type used in the quenching experiments, namely, that it be quenched in oil but be removed just before it reaches the boiling point of water and immediately tempered. This is a useful expedient provided that the surface has not become effectively elastic at the temperature of withdrawal. If it has, permanent stress will develop just as if cooled to ordinary temperatures in oil but will, of course, be relieved somewhat by the tempering action on cooling from $100^{\circ} \mathrm{C}$. If the piece be withdrawn before the surface becomes effectively elastic, as it should be for maximum safety, it can be cooled safely to ordinary temperatures and need not be tempered promptly. This procedure has the advantage that high stress is not developed at any time. Evidently withdrawal at $100^{\circ} \mathrm{C}$. does not accomplish this unless $A r^{\prime \prime}$ is low.

The same result can be attained, perhaps more conveniently, in another way, namely, by quenching in hot oil. The effect of this expedient was indicated in the last section and illustrated by Figure 17. The figure shows that tensional stress below a minimum which causes cracks will be obtained over a longer range of sizes the hotter the oil, or that in some moderate size, say 1 inch, the stress is lowered by heating the oil. To test this deduction, 1-inch diameter cylinders of the $J$ steel were quenched in oil at 25,60 , and $100^{\circ}$ C., Table 7 . The specimens quenched in oil at 25 and $60^{\circ}$ C. cracked as quenched, but the crack was smaller for oil at 60 than at $25^{\circ} \mathrm{C}$. The specimen quenched in oil at $100^{\circ} \mathrm{C}$. did not crack as quenched but did so on deep etching. Evidently a somewhat higher temperature is desirable in this case, but it need not be as high as the temperature at which the steel becomes effectively elastic, estimated to be $150^{\circ} \mathrm{C}$. in this case.

Hot oil is not required nor even desirable for sections which will be under surface compression as quenched in cold oil, for heating the oil might then throw the surface stress into tension. A similar situa- 
tion is apt to be encountered in the case of hot water. In moderate and large sizes cold water produces compressional surface stress, so that heating the water may reverse the stress to tension and thus crack the steel. As there is besides a considerable loss in cooling power at high surface temperatures on heating water, hot water has little to recommend it as a quenching medium. Oil, however, maintains its cooling power both for high and low surface temperatures on heating. These observations on the effect of bath temperature on the cooling power of oil and water are plainly revealed by the quenching curves of Pilling and Lynch (17).

TABLE 7.-Observations on quenching cracks in 1-inch diameter cylinders \& inches long produced by miscellaneous treatments of several compositions

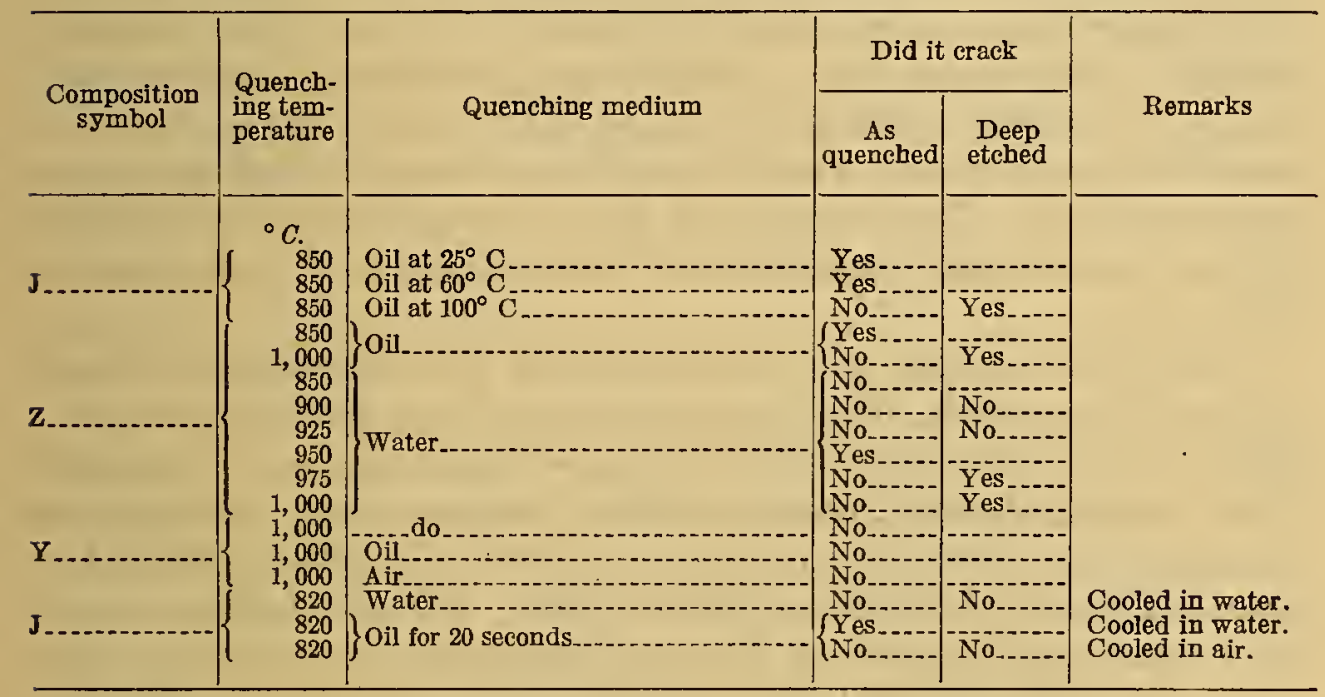

It would evidently be desirable to have a quenching medium which will cool as fast as oil, or faster, at high temperatures and much slower at low temperatures in order to avoid the undesirable, but necessary expedients mentioned above. This is, in fact, a description of the ideal quenching medium for the conditions in question. Such a medium does not appear to be available at present, but is, perhaps, worth looking for.

An appropriate objection to the treatments involving retarded cooling through $A r^{\prime \prime}$ is that hardness is sacrificed. The hardness is indeed less than when the steel is cooled continuously to ordinary temperatures as shown by Table 4 of reference (11), but the loss of hardness is compensated, at least in part, by less reduction in hardness on tempering. Here a low $A r^{\nu}$ is a decided advantage for the fast cooling can be maintained to a lower temperature with less transient tempering and loss of hardness during the retarded cooling.

Having thus found that certain undesirable thermal expedients are required to harden without cracking or warpage a certain range of 
sizes of a steel having the transformation characteristics defined by Figure $10(b)$, it remains to be determined whether or not these effects can be avoided by modification of the composition or other factors. Two possibilities are still open. First, the tensional surface stress can be reduced in magnitude and extent by reducing the linear expansion $\alpha_{2}\left(T_{\mathrm{m}}-T_{\mathrm{g}}\right)$, as illustrated by Figure 16 . Lowering the amount of dissolved carbon by means of the quenching temperature or composition will accomplish this, as shown by the expansion curves. These expedients, however, involve a considerable loss in hardness which it is our purpose to avoid.

The second possibility is to lower the temperature at which the steel becomes effectively elastic, $T_{\mathrm{s}}$. This reduces the tensional surface stress in a moderate size in the same way as raising the bath temperature but carries the additional advantage that transient tempering with its resultant hardness loss is correspondingly reduced. $T_{8}$ can be lowered either by increased alloy content or by increased quenching temperature when the carbide content is well above the eutectoid ratio. The lowering of $A r^{\prime \prime}$ by increasing the quenching temperature of a high carbon steel $Z$ from 850 to $1,000^{\circ} \mathrm{C}$. is shown in Figure 11.

Standard specimens of this composition were quenched in water and in oil from both these temperatures with the results recorded in Table 7. Quenched from $850^{\circ} \mathrm{C}$., the specimens cracked as quenched in oil, but not in water, just as did the $J$ composition. However, on raising the quenching temperature to $1,000^{\circ} \mathrm{C}$., neither cracked as quenched, though both did on deep etching. With the higher quenching temperature, conditions are more favorable to cracking in that $\alpha_{2}\left(T_{\mathrm{m}}-T_{\mathrm{s}}\right)$ is greater, but this unfavorable condition is more than overbalanced by the greatly reduced temperature drop coincident with lower $T_{\mathrm{s}}$. Because of the low drop, the surface is in tension after both oil and water quenching, but the tension is evidently fairly low.

Thus, in two ways, the conclusions from analysis alone that reducing $\left(T_{\mathrm{s}}-T_{\mathrm{o}}\right)$ will reduce tensional surface stress has been qualitatively confirmed. First, it was shown experimentally that raising $T_{0}$ with $T_{\mathrm{s}}$ constant reduces the cracking stress, and, second, that lowering $T_{\mathrm{s}}$ with $T_{\mathrm{o}}$ constant reduces the cracking stress. The continuous variation of the surface stress from tensional to compressional with increasing drop can also be established by varying $\left(T_{\mathrm{s}}-T_{\mathrm{o}}\right)$ through the quenching temperature. This is shown by increasing the temperature of quenching the $Z$ composition in water (Table 7). With a low quenching temperature, the temperature drop is large and the surface stress compression. No cracks are then observed as quenched or on deep etching. As the quenching temperature is raised, the drop is lowered to the region of maximum tensional surface stress 
and the piece cracks as quenched from $950^{\circ} \mathrm{C}$. For higher quenching temperatures, 975 and $1,000^{\circ} \mathrm{C}$., the piece cracked only on deep etching showing a reduction in the tensional stress at the surface.

The conclusion was also drawn from the stress equations that a high $A r^{\prime \prime}$ reduces the possibility of cracking. It has already been found that when $A r^{\prime \prime}$ is raised by lowering the quenching temperature cracking is reduced. Quenching specimens of the $Y$ composition which has a high $A r^{\prime \prime}$ also confirms this (Table 7). That these experiments gave results in the direction indicated by analysis is good evidence of the soundness of the fundamental conceptions advanced.

A simple experiment is strikingly confirmatory of the most important single conception, namely, that deep-seated cracking stress (surface tension) is produced only by a relatively small temperature drop. Three standard specimens of the $J$ composition were heated to $820^{\circ} \mathrm{C}$; the first was quenched in water until cold, the second in oil for 20 seconds and then in water, the third in oil for 20 seconds and then in air (Table 7). The first and third did not crack either before or after deep etching, but the second cracked as quenched. The temperature drop in the first case must have been very large, in the second considerably less, and in the third very small.

The close relation between the hardening transformation and the cracking stress established both analytically and experimentally indicates the importance of controlling this transformation. Cracks can not be consistently reproduced nor consistently avoided if this transformation varies among specimens from a particular steel. To control cracks successfully requires that they appear only under one known set of conditions which can be avoided. The expansion curves during the hardening transformation offer a means for checking the uniformity of a steel in this respect.

While only the permanent stresses in hardened steel have been considered and are certainly the major cause of quenching cracks, temporary stresses should not be ignored. Temporary stress exists only during the heating and cooling of elastic bodies. It is of importance in hardened steel because the steel is then quite brittle. Like glass, it can be heated fairly rapidly if heated uniformly, for this produces temporary compression at the surface, but it should not be cooled rapidly, for that produces temporary tension at the surface which is particularly undesirable when the surface is under permanent tension. Temporary surface tension may also produced during quenching in water after $A r^{\prime \prime}$ is completed if $A r^{\prime \prime}$ is high. A crack so produced would appear before the steel is cold and would be hard to distinguish from one produced by high permanent tension at the surface which may cause a crack to form before the steel is quite cold. 
Grinding may also produce temporary surface stress, but stress so produced will be compressional and hence not dangerous, provided that tempering does not occur. If, however, the steel is actually tempered under the grinding wheel, as has invariably been the case with grinding cracks observed by the writer, the metal will be reduced in volume, and thereby put under permanent tension which is the major source of cracks. Guillet and his associates (19) have recognized that tempering as revealed by loss in hardness is associated with grinding cracks, but attributes the cracks to plastic deformation during heating under the grinding wheel of areas which develop cracks. This explanation is, however, incompatible with the high elasticity of hardened tool steel under compression. Cracks might be produced by differential tempering on fast heating for tempering in the same manner. As hardened tool steel of low-alloy content is tempered, it first shrinks with increasing tempering temperature to between 150 and $180^{\circ} \mathrm{C}$., then expands slightly, and finally contracts rapidly. In order to minimize grinding cracks, the steel should evidently be tempered in the neighborhood of the first minimum before grinding. It will then take severe local heating to produce sufficient shrinkage to cause cracking. Grinding cracks are of little depth, so there should be no difficulty in distinguishing them from cracks primarily due to the quenching treatment.

While considerable space has been given to the discussion of means for preventing quenching cracks, the essential requirements can be put in a few words. First, find the dangerous range of sizes for the available quenching media and the steel and quenching temperature in question, then avoid it either by retarded cooling through $A r^{\prime \prime}$ or by a very fast quench. The first condition can be conveniently obtained by quenching in hot oil and the second by quenching in water. If the piece is of complicated shape, the latter treatment is precluded; if of carbon tool steel or a steel of very low-alloy content, the second is precluded. From this brief statement it is not to be inferred, however, that the problem is as simple as stated, for there are many variables which must be controlled by both the steel maker and the hardener if consistent results are to be attained.

\section{SUMMARY}

Cracks found in unground pieces of hardened tool steel were classified as local or deep-seated, depending on whether they were associated with sharp changes in contour or with the principal axes of the piece. The latter type was investigated experimentally, using solid cylinders of a particular oil-hardening steel. The following results were obtained on continuous quenching with agitation until the specimens reached the bath temperature: 
1. Cracks occurred on oil quenching cylinders between $3 / 4$ and $11 / 2$ inches diameter.

2. Cracks occurred with water quenching for diameters between $1 / 4$ and $1 / 2$ inch.

3. All the cracks were caused by tangential stress which was tensional at the surface.

4. The cracks did not develop to visual size until after the specimens reached ordinary temperatures throughout.

5. Cracks rarely occur when the surface is under compression, but when they do, they start at the center where the stress is tensional.

Measurements of the axial stresses in quenched cylinders by the method of Heyn showed that the stress at the surface is compressional when a deep-hardening steel of moderate or large size, 1 inch diameter or over, is quenched in water, or when only the surface hardened as on water quenching cylinders of carbon tool steel over $1 / 2$ inch in diameter. The surface stress in the latter case is higher than in the former.

From previous studies of stress generation in cooling glass and steel it is shown that the permanent stresses described above are produced by plastic deformation during quenching. Permanent tensional stress at the surface can be generated by cooling only when the steel is plastically deformed while the coefficient of expansion is negative. As this expansion during cooling is the chief cause of quenching cracks, its characteristics were studied experimentally.

Expansion measurements were made during cooling in an air stream after quenching deep-hardening steels in oil and removing the specimen before the $A r$ " transformation began. From the curves so obtained the following conclusions were drawn:

1. The transformation $A r^{\prime \prime}$ occurs in tool steels at a relatively low temperature accompanied by an expansion from a minimum volume to a volume greater than the original as annealed.

2. The contraction preceding $A r^{\prime \prime}$ is given approximately by the coefficient of expansion of austenite above the critical range.

3. The expansion during the initial stages of $A r^{\prime \prime}$ is approximately linear and the coefficient greater than that of austenite.

4. After the linear expansion the steel continues to expand, but at a decreasing rate.

5. The nonlinear expansion is attributed to the lowering of $A r^{\prime \prime}$ by pressure developed in the microscopic units after the formation of a continuous martensite network.

6. The steel is effectively plastic until the end of linear expansion.

7. The magnitude of the total expansion, as a rule, increases as $A r^{\prime \prime}$ is lowered.

8. Raising the quenching temperature lowers $A r^{\prime \prime}$ when there is an excess of carbide present. 
This information is adequate for the estimation of permanent stresses provided the temperature distribution is known in a simple shape when its surface has just become effectively elastic; that is, has reached the temperature $T_{s}$ of the end of linear expression. From the mathematical theory of heat conduction relations between the five chief variables determining the temperature distribution in a cylinder during cooling were derived and expressed in the form of curves. The temperature difference between the center and surface, called the temperature drop, was taken as a convenient measure of the temperature distribution. When small, the temperature drop is directly proportional to the surface temperature minus the bath temperature for a given bath and diameter of specimen. The temperature then varies approximately as the inverse of the square of the distance from the center.

Assuming certain ideal conditions, equations were derived for the stress within solid cylinders of full-hardening steel in terms of the temperature drop $\left(T_{\mathrm{o}}-T_{\mathrm{s}}\right)$; the temperature at which the surface becomes effectively elastic, $T_{\mathrm{s}}$; the temperature of minimum expansion, $T_{\mathrm{m}}$; and the coefficients of expansion before and during the early stages of $A r^{\prime \prime}, \alpha_{1}$, and $\alpha_{2}$, respectively. From these equations the following conclusions were drawn:

1. The surface stress increases with the temperature drop from zero to a maximum in tension, then decreases, passing through zero into compression and increases to a very high value in compression.

2. The maximum tensional surface stress that can be dereloped and the range of temperature drops which produce tension at the surface are directly proportional to the linear expansion during $A r^{\prime \prime}$.

3. When the temperature drop is less than the temperature range of linear expansion-that is, when $\left(T_{\mathrm{c}}-T_{\mathrm{s}}\right)$ is less than $\left(T_{\mathrm{m}}-T_{\mathrm{s}}\right)-$ the tensional surface stress is directly proportional to the temperature drop, the coefficient of expansion $\alpha_{2}$ remaining unchanged.

4. The minimum tensional stress which will crack tool steel is very low in comparison with the compressional stress that the steel will withstand at the surface.

If it be recognized that the cracks obtained experimentally were all due to permanent tensional stress at the surface and that uniformly distributed compressional stress at the surface does not produce cracks, it will be seen that these conclusions are compatible in all essential respects with the results of the quenching experiments.

These conclusions suggested expedients for the avoidance of quenching cracks in compositions which harden completely with the quenching given or proposed. In pieces of simple shape a condition which produces cracks can be avoided by using either a much more rapid quenching medium or by retarded cooling through the hardening 
transformation. This can be conveniently accomplished by quenching in water or hot oil, respectively. If the piece is of complicated shape, the treatment adopted must be of the latter type. Obviously, the choice is restricted by the composition of the steel. For example, if a complicated section of carbon tool steel cracks on water quenching, oil quenching can not be substituted without change of composition.

The tendency of a steel to crack can also be reduced by modifications of the quenching temperature, composition or both which either raise $A r^{\prime \prime}$ so that the linear expansion $\alpha_{2}\left(T_{\mathrm{m}}-T_{\mathrm{s}}\right)$ is small, or lower $A r^{\prime \prime}$ so that it is barely completed at ordinary temperatures. The first expedient can not be applied without sacrifice of hardness, so the second appears to be the most practicable.

Certain incidental causes of cracks should be recognized in order to avoid confusing them with the type investigated here. One is temporary tensional surface stress which is present during the cooling of pieces from the tempering bath and during quenching after $A r^{\prime \prime}$ is passed when $A r^{\prime \prime}$ is much above the bath temperature. Another is permanent tensional stress produced at the surface of hardened tool steel when the surface is tempered beyond the interior as may occur on surface grinding. The first-mentioned causes of cracks are probably rare, but the latter are not. Grinding cracks can, however, be definitely distinguished from deep-seated cracks by their lack of depth.

In conclusion, the writer wishes to acknowledge material aid in this work from several steel makers who supplied the steels used, from the Brown \& Sharpe Manufacturing Co., who contributed machine work, and from the gauge section of the Bureau of Standards which made the length measurements before and after taking expansion curves, and for the stress measurements by the Heyn method.

This paper developed from an investigation of gauge steels under the auspices of the Gauge Steel Committee for which work these accommodations were offered.

S. S. Kingsbury assisted in much of the experimental work presented.

\section{BIBLIOGRAPHY}

1. S. N. Brayshaw, "The prevention of hardening cracks and the effect of controlling recalescence, Jour. Iron and Steel Inst., 103, p. 131; 1921.

2. C. Benedicks, "Correspondence on Brayshaw's paper," Jour. Iron and Steel Inst., 103, p. 237; 1921.

3. A. Portevin, "Experimental contribution to the study of hardening cracks in shells," Rev. de Mét. 19, p. 267; 1922.

4. Schwinning, "Correspondence on Brayshaw's paper," Jour. Iron and Steel Inst., 103, p. 230; 1921. 
5. T. McL. Jasper, "Calculation of quenching stresses in hardened steel," Engn., 118, p. 343; 1924.

6. K. Honda, "On the cause of quenching cracks," Jour. Iron and Steel Inst., 103, p. $251 ; 1921$.

7. A. McCance, "Correspondence on Brayshaw's paper," Jour. Iron and Steel Inst., 103, p. 267; 1921.

8. E. Heyn, "Materialienkunde für den Maschinenbau," II A-1912, p. 331, and following.

9. G. Masing, "On the theory of thermal stresses," Zeit.f. Tech. Phys., 3, p. 167; 1922.

10. L. H. Adams and E. D. Willamson, "The annealing of glass," Jour. Frank. Inst., 190, pp. 597, 835; 1920.

11. H. Scott, "Quenching properties of glycerin and its water solutions," Trans. Am. Soc. for Steel Treating, 6, p. 13; 1924.

12. P. Chevenard, "Recording differential dilatometer-Description and first applications," Rév. de Met., 14, p. 610; 1917.

13. W. Souder and P. Hidnert, Thermal Expansion of a Few Steels, B. S. Sci. Papers No. 433.

14. R. P. Neville and J. R. Cain, The Preparation and Properties of Pure Iron Alloys: I. Effect of Carbon and Manganese on the Mechanical Properties of Iron, B. S. Sci. Papers No. 453.

15. H. S. Rawdon and S. Epstein, Structure of Martensitic Carbon Steels and Changes in Microstructure which Occur upon Tempering, B. S. Sci. Papers No. 452.

16. K. Heindlhofer, "Quenching: A mathematical study of various hypothesis on rapid cooling," Phys. Rev. N. S., 20, p. 221; 1922.

17. N. B. Pilling and T. D. Lynch, "Cooling properties of quenching liquids," Trans. Amer. Inst. of Mining and Met. Engrs., 62, p. 665; 1920.

18. Report of subcommittee on tool steel, Trans. Am. Soc. for Steel Treating, 6, p. $649 ; 1924$.

19. L. Guillet, J. Galibourg, and P. Beuret, "Les Criques de Rectification," Rev. de Mét. 18, p. 213; 1921.

WASHINGTON, May 14, 1925. 